\title{
On Nonlinear Dynamics of Predator-Prey Models with Discrete Delay*
}

\author{
S. $\operatorname{Ruan}^{\dagger}$ \\ Department of Mathematics, University of Miami, Coral Gables, FL 33124-4250, USA
}

\begin{abstract}
In this survey, we briefly review some of our recent studies on predator-prey models with discrete delay. We first study the distribution of zeros of a second degree transcendental polynomial. Then we apply the general results on the distribution of zeros of the second degree transcendental polynomial to various predator-prey models with discrete delay, including Kolmogorov-type predator-prey models, generalized Gause-type predator-prey models with harvesting, etc. Bogdanov-Takens bifurcations in delayed predator-prey models with nonmonotone functional response and in delayed predator-prey model with predator harvesting are also introduced.
\end{abstract}

Key words: predator-prey model, time delay, harvesting, stability, bifurcation AMS subject classification: $34 \mathrm{~K} 18,92 \mathrm{D} 25$

\section{Contents}

1. Introduction 141

1.1. Lotka-Volterra Predator-Prey Models with Discrete Delay . . . . . . . . . . . . . . 142

1.2. Non-Kolmogorov-Type Models . . . . . . . . . . . . . . . . . . . . . 143

1.3. Gause-Type Predator-Prey Systems with Discrete Delay and Harvesting . . . . . . 144

1.4. A Delayed Predator-Prey System with Nonmonotonic Functional Response . . . 145

*This survey is based on the lecture notes distributed at the Autumn School on "Delay Differential Equations and Applications", Marrakech, Morocco, September 9 - 21, 2002 and in the Department of Applied Mathematics at Shanghai Jiaotong University, Shanghai, China, December 27, 2007 - January 13, 2008.

${ }^{\dagger}$ Research was partially supported by NSF grant DMS-0715772. E-mail: ruan@math.miami.edu 
2. Stability of Delay Equations and Zeros of Transcendental Polynomials 146

2.1. Absolute and Conditional Stability . . . . . . . . . . . . . . . . . 146

2.2. Zeros of Transcendental Functions . . . . . . . . . . . . . . . . . 147

2.3. A Second Degree Transcendental Polynomial . . . . . . . . . . . . . . . . . 148

3. Kolmogorov-type Predator-Prey Systems with Discrete Delay 151

3.1. Delayed Inter-specific Interactions . . . . . . . . . . . . . . . . . 151

3.2. Delayed Predator Response . . . . . . . . . . . . . . . . . . . . 153

3.3. Delayed Prey Specific Growth . . . . . . . . . . . . . . . . 155

4. A Delayed Predator-Prey System with Nonmonotonic Functional Response 159

4.1. Hopf Bifurcation . . . . . . . . . . . . . . . . . . . 159

4.2. Bogdanov-Takens Bifurcation . . . . . . . . . . . . . . 164

5. Predator-Prey Models with Delay and Harvesting 169

5.1. Gause Models with Prey Harvesting and Delay in the Predator Response . . . . . . 169

5.2. Gause Models with Prey Harvesting and Delayed Prey Specific Growth . . . . . . 172

5.3. Wangersky-Cunningham Model with Prey Harvesting . . . . . . . . . . . . . . . 174

5.4. Predator Harvesting and Delayed Predator Response . . . . . . . . . . . . 177

$\begin{array}{ll}\text { 6. Discussion } & 180\end{array}$

\section{Introduction}

Predator-prey interaction is the fundamental structure in population dynamics. Understanding the dynamics of predator-prey models will be very helpful for investigating multiple species interactions. Delayed predator-prey models were first proposed by Volterra [83, 84] in 1925 to study fish population under harvesting. Since then delayed differential equations have been extensively used to model population dynamics, including predator-prey interactions. We refer to the monographs of Cushing [24], Gopalsamy [44], Kuang [56] and MacDonald [65] for general delayed biological systems. In general, delay differential equations exhibit much more complicated dynamics than ordinary differential equations since a time delay could cause a stable equilibrium to become unstable and induce bifurcations.

The original delayed predator-prey models proposed by Volterra $[83,84]$ are described by integrodifferential equations, such delays are also called distributed delays (Cushing [24] and MacDonald [65]), and discrete delays are special cases of the distributed delays when the kernels are taken as delta functions. However, predator-prey models with discrete delays have different features and can exhibit more complex dynamical behaviors, such as the existence of multiple equilibria, Hopf bifurcation, Bogdanov-Takens bifurcation, and even chaos (see, for example, Xiao and Ruan [92], Nakaoka et al. [69], etc.).

Recently, in a book chapter [75], I reviewed some basic single specie models described by (both discrete and distributed) delay differential equations. The purpose of this survey differs from that 
chapter. In this article, I will mainly review recent results obtained by myself and my collaborators on predator-prey models with discrete delays.

The main ideas are as follows. We first study the distribution of zeros of a second degree transcendental polynomial since for most predator-prey models with discrete delay the characteristic equation of the linearized system is a second degree transcendental equation. Applying the general results on the distribution of zeros of the second degree transcendental polynomial to various models, we find out that some predator-prey models with discrete delay (such as the generalized Gausetype predator-prey model with delayed predator response (Ruan [74])) is conditionally stable when the delay is less than a critical value, loses stability and undergoes a Hopf bifurcation when the delay passes through the critical value. Some other models (such as the May-type predator-prey model with delayed prey specific growth (Ruan [74])) can switch their stability when the delay takes different critical values. Secondly, thanks to the theories developed by Faria and Magalhãles $[32,34]$, we can show that some delayed predator-prey models (such as the delayed predator-prey model with nonmonotone functional response (Xiao and Ruan [92]) and the delayed predator-prey model with predator harvesting (Xia et al. [89])) can exhibit Bogdanov-Takens bifurcations.

We first review some well-known delayed predator-prey models. The presentation is basically taken from Ruan [74].

\subsection{Lotka-Volterra Predator-Prey Models with Discrete Delay}

Let $x(t)$ and $y(t)$ denote the population density of prey and predator at time $t$, respectively. The first predator-prey model with (distributed) delay was proposed by Volterra [84]. The modified version (see Brelot [17]) has the form

$$
\begin{aligned}
& \dot{x}(t)=x(t)\left[r_{1}-a_{11} x(t)-a_{12} \int_{-\infty}^{t} F(t-s) y(s) d s\right], \\
& \dot{y}(t)=y(t)\left[-r_{2}+a_{21} \int_{-\infty}^{t} G(t-s) x(s) d s-a_{22} y(t)\right],
\end{aligned}
$$

where $r_{1}>0$ is the growth rate of the prey in the absence of predators, $a_{11}>0$ denotes the self-regulation constant of the prey, $a_{12}>0$ describes the predation of the prey by predators, $r_{2}>0$ is the death rate of predators in the absence of the prey, $a_{21}>0$ is the conversion rate for the predators, and $a_{22} \geq 0$ describes the intraspecific competition among predators. $F$ and $G$ are nonnegative continuous delay kernels defined and integrable on $[0, \infty)$, which weight the contribution of the predation occurred in the past to the change rate of the prey and predators, respectively. Detailed study on stability and bifurcation of system (1.1) can be found in Cushing [24]. When $F(t-s)=\delta\left(t-s-\tau_{1}\right), G(t-s)=\delta\left(t-s-\tau_{2}\right)$, where $\delta$ is the Delta function, $\tau_{1}$ and $\tau_{2}$ are positive constants, system (1.1) reduces to the following Lotka-Volterra predator-prey model with two discrete delays

$$
\begin{aligned}
& \dot{x}(t)=x(t)\left[r_{1}-a_{11} x(t)-a_{12} y\left(t-\tau_{1}\right)\right], \\
& \dot{y}(t)=y(t)\left[-r_{2}+a_{21} x\left(t-\tau_{2}\right)-a_{22} y(t)\right] .
\end{aligned}
$$


When $F(t-s)=\delta(t-s)$ and $a_{22}=0$, system (1.1) becomes the following model

$$
\begin{aligned}
& \dot{x}(t)=x(t)\left[r_{1}-a_{11} x(t)-a_{12} y(t)\right], \\
& \dot{y}(t)=y(t)\left[-r_{2}+a_{21} \int_{-\infty}^{t} G(t-s) x(s) d s\right],
\end{aligned}
$$

which has been studied extensively (see Cushing [24], MacDonald [65], Dai [28], Farkas et al. [35], Stépán [81], etc.) and it has been shown that the time delay in (1.3) will destabilize the otherwise stable equilibrium and cause fluctuations in the populations via Hopf bifurcations. The discrete delay version of system (1.3) is

$$
\begin{aligned}
& \dot{x}(t)=x(t)\left[r_{1}-a_{11} x(t)-a_{12} y(t)\right], \\
& \dot{y}(t)=y(t)\left[-r_{2}+a_{21} x(t-\tau)\right],
\end{aligned}
$$

which has been considered in Kuang [56] and Beretta and Kuang [7].

Assuming that in the absence of predators the prey's growth is governed by delayed logistic equation, May [64] proposed the predator-prey system

$$
\begin{aligned}
& \dot{x}(t)=x(t)\left[r_{1}-a_{11} \int_{-\infty}^{t} F(t-s) x(s) d s-a_{12} y(t)\right], \\
& \dot{y}(t)=y(t)\left[-r_{2}+a_{21} x(t)-a_{22} y(t)\right] .
\end{aligned}
$$

Hassard et al. [48] provided detailed bifurcation analysis of system (1.5) and showed that system (1.5) exhibits Hopf bifurcation when the time delay passes through a critical value. May [64] gave a very brief discussion about the following discrete delay version of system (1.5)

$$
\begin{aligned}
& \dot{x}(t)=x(t)\left[r_{1}-a_{11} x(t-\tau)-a_{12} y(t)\right], \\
& \dot{y}(t)=y(t)\left[-r_{2}+a_{21} x(t)-a_{22} y(t)\right] .
\end{aligned}
$$

Recall that the system of ordinary differential equations

$$
\begin{aligned}
& \dot{x}(t)=x(t) f(x(t), y(t)), \\
& \dot{y}(t)=y(t) g(x(t), y(t))
\end{aligned}
$$

is called a Kolmogorov-type predator-prey system if $\frac{\partial f}{\partial y}<0$ and $\frac{\partial g}{\partial x}>0$. We can see that systems (1.2), (1.4) and (1.6) are of Kolmogorov-type predator-prey models. In [74], we considered three classes of more general Kolmogorov-type predator-prey models with discrete delay, which have systems (1.2), (1.4), and (1.6) as special cases, and studied absolute stability, conditional stability, and bifurcations in these systems.

\subsection{Non-Kolmogorov-Type Models}

Bartlett [4] and Wangersky and Cunningham [86] were the first to incorporate discrete delays into predator-prey models. Their models are not of the Kolmogorov-type. Bartlett assumed that there 
is a time lag $\tau_{1}$ in the growth to maturity of the prey, and there is a lag $\tau_{2}$ in the growth to maturity of the predators. He modified the Lotka-Volterra system into the form:

$$
\begin{aligned}
& \dot{x}(t)=r_{1} x\left(t-\tau_{1}\right)-a_{12} x(t) y(t), \\
& \dot{y}(t)=-r_{2} y(t)+a_{21} x\left(t-\tau_{2}\right) y\left(t-\tau_{2}\right) .
\end{aligned}
$$

Wangersky and Cunningham's model takes the form

$$
\begin{aligned}
& \dot{x}(t)=x(t)\left[r_{1}-a_{11} x(t)-a_{12} y(t)\right], \\
& \dot{y}(t)=-r_{2} y(t)+a_{21} x(t-\tau) y(t-\tau) .
\end{aligned}
$$

This model assumes that a duration of $\tau$ time units elapses when an individual prey is killed and the moment when the corresponding addition is made to the predator population. Wangersky and Cunningham [86] briefly analyzed their model (1.9), Goel et al. [41] pointed out that there are problems in Wangersky and Cunningham's analysis and reconsidered the model, but their own analysis is also incomplete. Some special cases of Bartlett's model (1.8) when $\tau_{1}=0$, or $\tau_{2}=0$, or $\tau_{1}=\tau_{2}$ have been studied by Hastings [49], Nunney [70, 71], Ma [61], Yan and Li [93], etc. Local stability analysis of Bartlett's model (1.8) was carried out in Hastings [50]. See also Ross [73] for a model where a delay appears only in $y(t)$ in the second equation.

\subsection{Gause-Type Predator-Prey Systems with Discrete Delay and Harvesting}

Time delays can be incorporated into the generalized Gause-type predator-prey model (Freedman [36]) in three different ways. (a) A time delay $\tau$ in the prey specific growth term $g(x(t))$, that is,

$$
\begin{aligned}
& \dot{x}(t)=x(t) g(x(t-\tau))-y(t) p(x(t)), \\
& \dot{y}(t)=y(t)[-d+\mu p(x(t))],
\end{aligned}
$$

where $g(x)$ represents the specific growth rate of the prey in the absence of predation and is assumed to satisfy

$$
g(0)>0, g(K)=0, g^{\prime}(x) \leq 0 \text { for } x>0 .
$$

$p(x)$ denotes the predator response function and is assumed to satisfy

$$
p(0)=0, p(x)>0 \text { for } x>0 .
$$

System (1.10) is proposed based on the assumption that in the absence of predators the prey satisfies the delayed logistic equation. We refer to May [61] for some discussion and analysis about (1.10) and its variants. (b) A time delay $\tau$ in the predator response term $p(x(t))$ in the predator equation, that is,

$$
\begin{aligned}
& \dot{x}(t)=x(t) g(x(t))-y(t) p(x(t)), \\
& \dot{y}(t)=y(t)[-d+\mu p(x(t-\tau))] .
\end{aligned}
$$

The delay in system (1.11) can be regarded as a gestation period or reaction time of the predators. System (1.11) has been studied extensively, we refer to Kuang [56], Beretta and Kuang [7] and the 
references therein. (c) A time delay $\tau$ in the interaction term $y(t) p(x(t))$ of the predator equation, that is,

$$
\begin{aligned}
& \dot{x}(t)=x(t) g(x(t))-y(t) p(x(t)), \\
& \dot{y}(t)=-d y(t)+\mu y(t-\tau) p(x(t-\tau)) .
\end{aligned}
$$

System (1.12) assumes that the change rate of predators depends on the number of prey and of predators present at some previous time. The well-known Wangersky-Cunningham model (1.9) is such a model. In section 3, we will present detailed results from Ruan [74] on stability and bifurcation about models (1.10), (1.11) and (1.12).

Predator-prey systems with constant-rate harvesting have been studied extensively and very complex dynamical behaviors, such as the existence of multiple equilibria, homoclinic loop, Hopf bifurcation and Bogdanov-Takens bifurcation, have been observed, see, for example, Brauer and Soudack [14, 15, 16], Beddington and Cooke [5], Dai and Tang [27], Hogarth et al. [53], Myerscough et al. [68], Xiao and Ruan [91]. Brauer [11] was the first to consider the combined effects of time delay and constant harvesting on predator-prey models. Martin and Ruan [63] and Xia et al. [89] studied the combined effects of constant-rate harvesting and delay on the dynamics of some predator-prey systems. Namely, (a) a generalized Gause-type predator-prey model with prey harvesting and a time delay in the predator response function (Martin and Ruan [63]). (b) A generalized Gause-type predator-prey model with prey harvesting and a time delay in the prey specific growth term will be analyzed (Martin and Ruan [63]). (c) The Wangersky-Cunningham predator-prey model with prey harvesting (Martin and Ruan [63]). (d) A generalized Gause-type predator-prey model with predator harvesting and a time delay in the predator response function (Xia et al. [89]). Their results will be discussed in section 5 .

\subsection{A Delayed Predator-Prey System with Nonmonotonic Functional Re- sponse}

In general the functional response $p(x)$ is a monotone function. However, there are experiments in microbial dynamics that indicate that nonmonotonic responses occur at the microbial level: when the nutrient concentration reaches a high level an inhibitory effect on the specific growth rate may occur. This is often seen when micro-organisms are used for waste decomposition or for water purification (see Andrews [1], Sokol and Howell [78], Bush and Cook [18]). In population dynamics, nonmonotonic functional response occurs when the prey exhibits group defense (Freedman and Wolkowicz [40], Wolkowicz [87]), the phenomenon whereby predation is decreased, or even prevented altogether, due to the increased ability of the prey to better defend or disguise themselves when their numbers are large enough.

Based on some experimental data, Caperon [20] observed that there is a time delay between the changes in substrate concentration and the corresponding changes in the bacterial growth rate. Following Caperon's observation, Bush and Cook [18] allowed the growth rate of microorganism to depend on the substrate concentrations $\tau$ units of time earlier and proposed a system of delayed 
differential equations of the form:

$$
\begin{aligned}
& \dot{x}(t)=r x(t)\left(1-\frac{x(t)}{K}\right)-\frac{x(t) y(t)}{a+x^{2}(t)}, \\
& \dot{y}(t)=y(t)\left[\frac{\mu x(t-\tau)}{a+x^{2}(t-\tau)}-D\right],
\end{aligned}
$$

where $r, K, a, \mu, D$, and $\tau$ are positive constants.

Recently, Ruan and Xiao [77] studied the dynamics of system (1.13) with $\tau=0$ and found that the model undergoes a series of bifurcations including saddle-node bifurcation, Hopf bifurcations, and homoclinic bifurcation. Xiao and Ruan [92] carried out a bifurcation analysis of delayed system (1.13) and showed that there is a Bogdanov-Takens singularity for any time delay value. Their results will be introduced in section 4 .

\section{Stability of Delay Equations and Zeros of Transcendental Polynomials}

\subsection{Absolute and Conditional Stability}

In this section, we review some results on the stability of delay differential equations and the distribution of zeros of transcendental polynomials. The presentation here is mainly adapted from Ruan [74] and Ruan and Wei [76].

Consider the following general nonlinear delay differential system

$$
\dot{x}(t)=f\left(x(t), x\left(t-\tau_{1}\right), \cdots, x\left(t-\tau_{m}\right)\right),
$$

where $x \in R^{n}, \tau_{j} \geq 0(1 \leq j \leq m)$ are constants, $f: R^{n} \times C^{m} \rightarrow R^{n}$ is assumed to be smooth enough to guarantee the existence and uniqueness of solutions to (2.1) under the initial value condition (Bellman and Cooke [6] and Hale and Verduyn Lunel [47])

$$
x(\theta)=\phi(\theta), \quad \theta \in[-\tau, 0],
$$

where $C=C\left([-\tau, 0], R^{n}\right), \tau=\max _{1 \leq i \leq m}\left\{\tau_{i}\right\}$. Suppose $f\left(x^{*}, x^{*}, \cdots, x^{*}\right)=0$, that is, $x=x^{*}$ is a steady state of system (2.1).

Definition 1. The steady state $x=x^{*}$ of system (2.1) is said to be absolutely stable (i.e., asymptotically stable independent of the delays) if it is asymptotically stable for all delays $\tau_{j} \geq 0(1 \leq$ $j \leq m) . x=x^{*}$ is said to be conditionally stable (i.e., asymptotically stable depending on the delays) if it is asymptotically stable for $\tau_{j}(1 \leq j \leq m)$ in some intervals, but not necessarily for all delays $\tau_{j} \geq 0(1 \leq j \leq m)$.

The linearized system of (2.1) at $x=x^{*}$ has the form:

$$
\dot{X}(t)=A_{0} X(t)+\sum_{j=1}^{m} A_{j} X\left(t-\tau_{j}\right),
$$


where $X \in R^{n}$, each $A_{j}(0 \leq j \leq m)$ is an $n \times n$ constant matrix. Thus, the steady state $x=x^{*}$ of system (2.1) is absolutely stable (conditionally stable) if the trivial solution of the linearized system (2.3) is absolutely stable (conditionally stable). Note that we are dealing with local stability of system (2.1).

The characteristic equation associated with system (2.3) takes the form:

$$
\operatorname{det}\left[\lambda I-A_{0}-\sum_{j=1}^{m} A_{j} e^{-\lambda \tau_{j}}\right]=0 \text {. }
$$

The transcendental equation (2.4) and its variants have been studied by many researchers, see Baptistini and Táboas [3], Bellman and Cooke [6], Boese [10], Brauer [13], Cooke and van den Driessche [23], Cooke and Grossman [22], Huang [54], Mahaffy [62], Ruan and Wei [76] and the references therein. The following result, which was proved by Chin [21] for $m=1$ and by Datko [29] and Hale et al. [46] for $m \geq 1$, gives necessary and sufficient conditions for the absolute stability of system (2.3).

Lemma 2. System (2.3) is absolutely stable if and only if

(i) $\operatorname{Re} \lambda\left(\sum_{j=0}^{m} A_{j}\right)<0$;

(ii) $\operatorname{det}\left[i \omega I-A_{0}-\sum_{j=1}^{m} A_{j} e^{-i \omega \tau_{j}}\right] \neq 0$ for all $\omega>0$.

Assumption (i) guarantees that system (2.3) with $\tau_{j}=0(1 \leq j \leq m)$ is asymptotically stable while assumption (ii) ensures that $i \omega$ is not a root of equation (2.4). Thus, roughly speaking, Lemma 2 says that the delay system (2.3) is absolutely stable if and only if the corresponding ODE system is asymptotically stable and the characteristic equation (2.4) has no purely imaginary roots.

Lemma 2 will be used to study stability and bifurcation in various delayed systems. The main idea is as follows. If assumption (ii) does not hold, that is, if the characteristic equation (2.4) has a pair of purely imaginary roots, say $\pm i \omega_{0}$, then system (2.3) is not absolutely stable but can be conditionally stable. Suppose $\omega_{0}$ is achieved when one of the delays, say $\tau_{1}$, reaches a value $\tau_{1}^{0}$. When $\tau_{1}<\tau_{1}^{0}$ the real parts of all roots of the characteristic equation (2.4) still remain negative and system (2.3) is conditionally stable. When $\tau_{1}=\tau_{1}^{0}$, the characteristic equation (2.4) has a pair of purely imaginary roots $\pm i \omega_{0}$ and system (2.3) loses its stability. By Rouché's theorem (Dieudonné [30]) and continuity, if the transversality condition holds at $\tau_{1}=\tau_{1}^{0}$, then when $\tau_{1}>$ $\tau_{1}^{0}$ the characteristic equation (2.4) will have at least one root with positive real part and system (2.3) becomes unstable. Moreover, Hopf bifurcation occurs, that is, a family of periodic solutions bifurcates from the steady state as $\tau_{1}$ passes through the critical value $\tau_{1}^{0}$.

\subsection{Zeros of Transcendental Functions}

In this section, we state some basic results on zeros of some transcendental functions (see Ruan and Wei [76]). 
Theorem 3. Suppose that $B \subset \mathbf{R}^{n}$ is an open connected set, $h(\lambda, \mu)$ is continuous in $(\lambda, \mu) \in$ $\mathbf{C} \times B$ and analytic in $\lambda \in \mathbf{C}$, and the zeros of $h(\lambda, \mu)$ in the right half plane

$$
\{\lambda \in \mathbf{C}: \operatorname{Re} \lambda \geq 0\}
$$

are uniformly bounded. If for any $\mu \in B_{1} \subset B$, where $B_{1}$ is a bounded, closed, and connected set, $h(\lambda, \mu)$ has no zeros on the imaginary axis, then the sum of the orders of the zeros of $h(\lambda, \mu)$ in the open right half plane $(\operatorname{Re} \lambda>0)$ is a fixed number for $B_{1}$, that is, it is independent of the parameter $\mu \in B_{1}$.

Now we apply Theorem 3 to a general exponential polynomial

$$
\begin{aligned}
P\left(\lambda, e^{-\lambda \tau_{1}}, \cdots, e^{-\lambda \tau_{m}}\right) & \\
= & \lambda^{n}+p_{1}^{(0)} \lambda^{n-1}+\cdots+p_{n-1}^{(0)} \lambda+p_{n}^{(0)} \\
& +\left[p_{1}^{(1)} \lambda^{n-1}+\cdots+p_{n-1}^{(1)} \lambda+p_{n}^{(1)}\right] e^{-\lambda \tau_{1}}+\cdots \\
& +\left[p_{1}^{(m)} \lambda^{n-1}+\cdots+p_{n-1}^{(m)} \lambda+p_{n}^{(m)}\right] e^{-\lambda \tau_{m}},
\end{aligned}
$$

where $\tau_{i} \geq 0(i=1,2, \ldots, m)$ and $p_{j}^{(i)}(i=0,1, \cdots, m ; j=1,2, \cdots, n)$ are constants.

Corollary 4. As $\left(\tau_{1}, \tau_{2}, \cdots, \tau_{m}\right)$ vary, the sum of the orders of the zeros of $P\left(\lambda, e^{-\lambda \tau_{1}}, \cdots, e^{-\lambda \tau_{m}}\right)$ in the open right half plane can change only if a zero appears on or crosses the imaginary axis.

\subsection{A Second Degree Transcendental Polynomial}

For most predator-prey systems with a discrete delay, the characteristic equation of the linearized system at a steady state is a second degree transcendental polynomial equation of the form:

$$
\lambda^{2}+p \lambda+r+(s \lambda+q) e^{-\lambda \tau}=0,
$$

where $p, r, q, s$ are real numbers. It is known that the steady state is asymptotically stable if all roots of the characteristic equation (2.6) have negative real parts. In this section, we introduce the results from Ruan [74] on the distribution of the roots of the characteristic equation (2.6).

When $\tau=0$, equation (2.6) becomes

$$
\lambda^{2}+(p+s) \lambda+(q+r)=0 .
$$

Assume that all roots of equation (2.7) have negative real parts, which is true if and only if

$\left(H_{1}\right) p+s>0$

$\left(H_{2}\right) q+r>0$.

We want to determine if the real part of some root increases to reach zero and eventually becomes positive as $\tau$ varies. If $i \omega$ is a root of equation (2.6), then

$$
-\omega^{2}+i p \omega+i s \omega(\cos \tau \omega-i \sin \tau \omega)+r+q(\cos \tau \omega-i \sin \tau \omega)=0 .
$$


Separating the real and imaginary parts, we have

$$
\begin{aligned}
-\omega^{2}+r & =-q \cos \tau \omega-q \omega \sin \tau \omega, \\
p \omega & =-q \omega \cos \tau \omega+q \sin \tau \omega .
\end{aligned}
$$

It follows that $\omega$ satisfies

$$
\omega^{4}-\left(s^{2}-p^{2}+2 r\right) \omega^{2}+\left(r^{2}-q^{2}\right)=0 .
$$

The two roots of equation (2.9) can be expressed as follows

$$
\omega_{ \pm}^{2}=\frac{1}{2}\left(s^{2}-p^{2}+2 r\right) \pm \frac{1}{2}\left[\left(s^{2}-p^{2}+2 r\right)^{2}-4\left(r^{2}-q^{2}\right)\right]^{\frac{1}{2}} .
$$

Thus, if

$\left(H_{3}\right) s^{2}-p^{2}+2 r<0$ and $r^{2}-q^{2}>0$ or $\left(s^{2}-p^{2}+2 r\right)^{2}<4\left(r^{2}-q^{2}\right)$,

then none of $\omega_{+}^{2}$ and $\omega_{-}^{2}$ is positive, that is, equation (2.9) does not have positive roots. Therefore, characteristic equation (2.6) does not have purely imaginary roots. Since $\left(H_{1}\right)$ and $\left(H_{2}\right)$ ensure that all roots of equation (2.7) have negative real parts, by Rouché's theorem, it follows that the roots of equation (2.6) have negative real parts too.

We have the following lemma (Ruan [74]).

Lemma 5. If $\left(H_{1}\right)-\left(H_{3}\right)$ hold, then all roots of equation (2.6) have negative real parts for all $\tau \geq 0$.

On the other hand, if

$\left(H_{4}\right) r^{2}-q^{2}<0$ or $s^{2}-p^{2}+2 r>0$ and $\left(s^{2}-p^{2}+2 r\right)^{2}=4\left(r^{2}-q^{2}\right)$,

then equation (2.9) has a positive root $\omega_{+}^{2}$. If

$\left(H_{5}\right) r^{2}-q^{2}>0, s^{2}-p^{2}+2 r>0$ and $\left(s^{2}-p^{2}+2 r\right)^{2}>4\left(r^{2}-q^{2}\right)$,

then equation (2.9) has two positive roots $\omega_{ \pm}^{2}$. In both cases, the characteristic equation (2.6) has purely imaginary roots when $\tau$ takes certain values. These critical values $\tau_{j}^{ \pm}$of $\tau$ can be determined from system (2.8), given by

$$
\tau_{j}^{ \pm}=\frac{1}{\omega_{ \pm}} \arccos \left\{\frac{q\left(\omega_{ \pm}^{2}-r\right)-p s \omega_{ \pm}^{2}}{s^{2} \omega_{ \pm}^{2}+q^{2}}\right\}+\frac{2 j \pi}{\omega_{ \pm}}, j=0,1,2, \cdots
$$

The above analysis can be summarized into the following lemma (Ruan [74]).

Lemma 6. (i) If $\left(H_{1}\right),\left(H_{2}\right)$ and $\left(H_{4}\right)$ hold and $\tau=\tau_{j}^{+}$, then equation (2.6) has a pair of purely imaginary roots $\pm i w_{+}$.

(ii) If $\left(H_{1}\right),\left(H_{2}\right)$ and $\left(H_{5}\right)$ hold and $\tau=\tau_{j}^{+}$( $\tau=\tau_{j}^{-}$respectively), then equation (2.6) has a pair of imaginary roots $\pm i w_{+}\left( \pm i w_{-}\right.$respectively). 
We would expect that the real part of some root to equation (2.6) becomes positive when $\tau>\tau_{j}^{+}$ and $\tau<\tau_{j}^{-}$. To see if it is the case, denote

$$
\lambda_{j}^{ \pm}=\alpha_{j}^{ \pm}(\tau)+i w_{j}^{ \pm}(\tau), \quad j=0,1,2, \cdots
$$

the root of equation (2.6) satisfying

$$
\alpha_{j}^{ \pm}\left(\tau_{j}^{ \pm}\right)=0, \quad w_{j}^{ \pm}\left(\tau_{j}^{ \pm}\right)=\omega_{ \pm} .
$$

We can verify that the following transversality conditions hold:

$$
\frac{d}{d \tau} \operatorname{Re} \lambda_{j}^{+}\left(\tau_{j}^{+}\right)>0, \quad \frac{d}{d \tau} \operatorname{Re} \lambda_{j}^{-}\left(\tau_{j}^{-}\right)<0 .
$$

It follows that $\tau_{j}^{ \pm}$are bifurcation values. Thus, we have the following theorem about the distribution of the characteristic roots of equation (2.6) (Ruan [74]).

Theorem 7. Let $\tau_{j}^{ \pm}(j=0,1,2, \cdots)$ be defined by (2.11).

(i) If $\left(H_{1}\right)-\left(H_{3}\right)$ hold, then all roots of equation (2.6) have negative real parts for all $\tau \geq 0$.

(ii) If $\left(H_{1}\right),\left(H_{2}\right)$ and $\left(H_{4}\right)$ hold, then when $\tau \in\left[0, \tau_{0}^{+}\right)$all roots of equation (2.6) have negative real parts, when $\tau=\tau_{0}^{+}$equation (2.6) has a pair of purely imaginary roots $\pm i \omega_{+}$, and when $\tau>\tau_{0}^{+}$equation (2.6) has at least one root with positive real part.

(iii) If $\left(H_{1}\right),\left(H_{2}\right)$ and $\left(H_{5}\right)$ hold, then there is a positive integer $k$ such that

$$
0<\tau_{0}^{+}<\tau_{0}^{-}<\tau_{1}^{+}<\cdots<\tau_{k-1}^{-}<\tau_{k}^{+}
$$

and there are $k$ switches from stability to instability to stability; that is, when

$$
\tau \in\left[0, \tau_{0}^{+}\right),\left(\tau_{0}^{-}, \tau_{1}^{+}\right), \cdots,\left(\tau_{k-1}^{-}, \tau_{k}^{+}\right),
$$

all roots of equation (2.6) have negative real parts, and when

$$
\tau \in\left[\tau_{0}^{+}, \tau_{0}^{-}\right),\left[\tau_{1}^{+}, \tau_{1}^{-}\right), \cdots,\left[\tau_{k-1}^{+}, \tau_{k-1}^{-}\right) \text {and } \tau>\tau_{k}^{+},
$$

equation (2.6) has at least one root with positive real part.

Remark 8. We should mention that the main part of Theorem 7 was obtained by Cooke and Grossman [22] in analyzing a general second order equation with delayed friction and restoring force. For other related work, see Baptistini and Táboas [3], Bellman and Cooke [6], Boese [10], Brauer [13], Cooke and van den Driessche [23], Cooke and Grossman [22], Huang [54], Mahaffy [62], etc. 


\section{Kolmogorov-type Predator-Prey Systems with Discrete De- lay}

In this section, we shall use the results in section 2 to study absolute stability, conditional stability and bifurcation of three classes of Kolmogorov-type predator-prey systems with discrete delay which have systems (1.2), (1.4) and (1.6) as special cases, respectively (see Ruan [74]).

\subsection{Delayed Inter-specific Interactions}

We first consider a Kolmogorov-type predator-prey model with discrete delays appearing in the inter-specific interaction terms of both equations:

$$
\begin{aligned}
& \dot{x}(t)=x(t) f\left(x(t), y\left(t-\tau_{1}\right)\right), \\
& \dot{y}(t)=y(t) g\left(x\left(t-\tau_{2}\right), y(t)\right),
\end{aligned}
$$

where $\tau_{i} \geq 0(i=1,2)$ is a constant. Denote $C=C([-\tau, 0], R)$, where $\tau=\max \left\{\tau_{1}, \tau_{2}\right\}$. Assume that $f: R \times C \rightarrow R$ and $g: C \times R \rightarrow R$ satisfy the following assumptions:

$\left(A_{1}\right)$ there exists a point $\left(x^{*}, y^{*}\right)$ with $x^{*}>0, y^{*}>0$ for which $f\left(x^{*}, y^{*}\right)=g\left(x^{*}, y^{*}\right)=0$;

$\left(A_{2}\right) f$ and $g$ are continuously differentiable such that

$$
\frac{\partial f}{\partial x}<0, \quad \frac{\partial f}{\partial y}<0, \quad \frac{\partial g}{\partial x}>0, \quad \frac{\partial g}{\partial y}<0 .
$$

Note that the assumptions in $\left(A_{2}\right)$ ensure that system (3.1) is a Kolmogorov-type predator-prey system. See also assumption $\left(A_{2}^{\prime}\right)$ in section 3.2. The initial values of system (3.1) are

$$
x(\theta)=\phi(\theta) \geq 0, \quad y(\theta)=\psi(\theta) \geq 0, \quad \theta \in[-\tau, 0],
$$

where $\phi$ and $\psi$ are continuous functions.

Assumption $\left(A_{1}\right)$ ensures that $\left(x^{*}, y^{*}\right)$ is a positive equilibrium of system (3.1).

Definition 9. The positive equilibrium $\left(x^{*}, y^{*}\right)$ is said to be asymptotically stable if there exists a $\delta>0$ such that

$$
\sup _{-\tau \leq \theta \leq 0}\left[\left|\phi(\theta)-x^{*}\right|+\left|\psi(\theta)-y^{*}\right|\right]<\delta
$$

implies

$$
\lim _{t \rightarrow \infty}(x(t), y(t))=\left(x^{*}, y^{*}\right),
$$

where $(x(t), y(t))$ is the solution of system (3.1) with initial values (3.2). 
The equilibrium $\left(x^{*}, y^{*}\right)$ of system (3.1) is asymptotically stable if the zero equilibrium $(0,0)$ of the linearized system at $\left(x^{*}, y^{*}\right)$ is asymptotically stable. Let

$$
X(t)=x(t)-x^{*}, \quad Y(t)=y(t)-y^{*} .
$$

Then the linearized system at $\left(x^{*}, y^{*}\right)$ is

$$
\begin{aligned}
& \dot{X}(t)=\left(a x^{*}\right) X(t)+\left(b x^{*}\right) Y\left(t-\tau_{1}\right), \\
& \dot{Y}(t)=\left(c y^{*}\right) X\left(t-\tau_{2}\right)+\left(d y^{*}\right) Y(t),
\end{aligned}
$$

where

$$
a=\frac{\partial f}{\partial x}\left(x^{*}, y^{*}\right), \quad b=\frac{\partial f}{\partial y}\left(x^{*}, y^{*}\right), \quad c=\frac{\partial g}{\partial x}\left(x^{*}, y^{*}\right), \quad d=\frac{\partial g}{\partial y}\left(x^{*}, y^{*}\right) .
$$

The characteristic equation is

$$
\lambda^{2}-\left(a x^{*}+d y^{*}\right) \lambda+a d x^{*} y^{*}-b c x^{*} y^{*} e^{-\lambda\left(\tau_{1}+\tau_{2}\right)}=0 .
$$

Let $\tau=\tau_{1}+\tau_{2}$. Then it can be written as

$$
\lambda^{2}+p \lambda+r+q e^{-\lambda \tau}=0,
$$

where

$$
p=-2\left(a x^{*}+d y^{*}\right)>0, \quad r=4 a d x^{*} y^{*}>0, \quad q=4 b c x^{*} y^{*}<0 .
$$

Equation (3.6) is a special case of equation (2.6) with $s=0$. Clearly, we can see that $\left(H_{1}\right)$ is satisfied. Since $a d-b c>0$, we have

$$
q+r=4(a d-b c) x^{*} y^{*}>0,
$$

which implies that $\left(H_{2}\right)$ is satisfied. We also have

$$
-p^{2}+2 r=-4\left(a^{2}\left(x^{*}\right)^{2}+d^{2}\left(y^{*}\right)^{2}\right)<0, r^{2}-q^{2}=16\left(x^{*}\right)^{2}\left(y^{*}\right)^{2}(a d+b c)(a d-b c) .
$$

So $\left(H_{3}\right)$ is satisfied if $a d+b c>0$. By Theorem 7, all roots of the characteristic equation (3.6) have negative real parts if and only if $a d+b c>0$. Therefore, we have the following theorem (Ruan [74]).

Theorem 10. Suppose that $f$ and $g$ satisfy the assumptions $\left(A_{1}\right)$ and $\left(A_{2}\right)$. Then the positive equilibrium $\left(x^{*}, y^{*}\right)$ of the delayed predator-prey system (3.1) is absolutely stable if and only if $a d+b c>0$.

Remark 11. System (3.1) with $\tau_{1}=\tau_{2}$ was studied by Gopalsamy [43] who showed that the delay is "harmless" in the sense that the positive steady state is asymptotically stable independent of the delay. Our result not only supports Gopalsamy's claim but also generalizes his to the case with two delays. 
Example 12. As an example, consider the Lotka-Volterra predator-prey model with two discrete delays:

$$
\begin{aligned}
& \dot{x}(t)=x(t)\left[r_{1}-a_{11} x(t)-a_{12} y\left(t-\tau_{1}\right)\right], \\
& \dot{y}(t)=y(t)\left[-r_{2}+a_{21} x\left(t-\tau_{2}\right)-a_{22} y(t)\right],
\end{aligned}
$$

where $r_{i}, a_{i j}(i, j=1,2)$ are positive constants. If

$$
r_{1} a_{21}-r_{2} a_{11}>0
$$

then system (3.7) has a positive equilibrium $E^{*}=\left(x^{*}, y^{*}\right)$, where

$$
x^{*}=\frac{r_{1} a_{22}+r_{2} a_{12}}{a_{11} a_{22}+a_{12} a_{21}}, \quad y^{*}=\frac{r_{1} a_{21}-r_{2} a_{11}}{a_{11} a_{22}+a_{12} a_{21}} .
$$

The condition $a d+b c>0$ becomes $a_{11} a_{22}-a_{12} a_{21}>0$. Thus, by Theorem 10 , we have the following result on the stability of $E^{*}=\left(x^{*}, y^{*}\right)$.

Corollary 13. If condition (3.8) is satisfied, i.e., if the positive equilibrium $E^{*}=\left(x^{*}, y^{*}\right)$ of system (3.7) exists, then it is absolutely stable if and only if $a_{11} a_{22}-a_{12} a_{21}>0$.

Remark 14. By the results in He [51] and Lu and Wang [60] we can see that the positive equilibrium $E^{*}=\left(x^{*}, y^{*}\right)$ of system (3.7) is indeed globally stable.

The above stability result depends on the assumption that $a_{11} a_{22}-a_{12} a_{21}>0$. If $a_{11} a_{22}-$ $a_{12} a_{21}<0$, then Theorem 7 can be used to show that when one of the delays varies, the positive equilibrium $E^{*}$ loses its stability and a Hopf bifurcation can occur. In fact, Faria [32] proved the following result (see also Song et al. [79]).

Corollary 15. If $a_{11} a_{22}-a_{12} a_{21}<0$ and $a_{11} a_{22} \neq 0$, then there is a critical value $\tau_{2,0}>0$, such that $E^{*}$ of system (3.7) is asymptotically stable when $\tau_{2}<\tau_{2,0}$ and unstable when $\tau_{2}>\tau_{2,0}$. A Hopf bifurcation exists at $E^{*}$ when $\tau_{2}=\tau_{2,0}$ in a two-dimensional stable local center manifold.

\subsection{Delayed Predator Response}

Next we consider a Kolmogorov-type predator-prey model with a discrete delay appearing in the inter-specific interaction term of the predator equation:

$$
\begin{aligned}
& \dot{x}(t)=x(t) f(x(t), y(t)), \\
& \dot{y}(t)=y(t) g(x(t-\tau), y(t)),
\end{aligned}
$$

where $\tau \geq 0$ is a constant, $C=C([-\tau, 0], R), f: R^{2} \rightarrow R$ and $g: C \times R \rightarrow R$ satisfy the assumptions $\left(A_{1}\right)$ and

$\left(A_{2}^{\prime}\right) f$ and $g$ are continuously differentiable such that

$$
\frac{\partial f}{\partial x}<0, \quad \frac{\partial f}{\partial y}<0, \quad \frac{\partial g}{\partial x}>0, \quad \frac{\partial g}{\partial y} \leq 0 .
$$


The initial values of system (3.10) are

$$
x(\theta)=\phi(\theta) \geq 0, \theta \in[-\tau, 0], \quad y(0)=y_{0} \geq 0 .
$$

By the assumption $\left(A_{1}\right),\left(x^{*}, y^{*}\right)$ is a positive equilibrium of system (3.10). The stability of $\left(x^{*}, y^{*}\right)$ can be defined analogously as in the previous model. Using (3.3), we have the linearized system:

$$
\begin{aligned}
& \dot{X}(t)=\left(a x^{*}\right) X(t)+\left(b x^{*}\right) Y(t), \\
& \dot{Y}(t)=\left(c y^{*}\right) X(t-\tau)+\left(d y^{*}\right) Y(t),
\end{aligned}
$$

where $a<0, b<0, c>0$ and $d \leq 0$ are defined in (3.5). The characteristic equation is

$$
\lambda^{2}-\left(a x^{*}+d y^{*}\right) \lambda+a d x^{*} y^{*}-b c x^{*} y^{*} e^{-\lambda \tau}=0 .
$$

Compared with (2.6), we have

$$
p=-\left(a x^{*}+d y^{*}\right)>0, r=a d x^{*} y^{*} \geq 0, s=0, q=-b c x^{*} y^{*}>0 .
$$

Hence, conditions $\left(H_{1}\right)$ and $\left(H_{2}\right)$ are satisfied. Also,

$$
-p^{2}+2 r=-\left(a x^{*}\right)^{2}-\left(b x^{*}\right)^{2}<0, r^{2}-q^{2}=(a d+b c)(a d-b c)\left(x^{*} y^{*}\right)^{2} .
$$

It follows that if $a d+b c>0$, then condition $\left(H_{3}\right)$ holds; if $a d+b c<0$, then conditions $\left(H_{4}\right)$ holds. Denote

$$
\omega_{+}=\left\{\frac{1}{2}\left(2 r-p^{2}\right)+\left[\frac{1}{4}\left(2 r-p^{2}\right)^{2}-\left(r^{2}-q^{2}\right)\right]^{\frac{1}{2}}\right\}^{\frac{1}{2}}
$$

and

$$
\tau_{j}^{+}=\frac{1}{\omega_{+}} \arccos \left\{\frac{\omega_{+}^{2}-r}{q}\right\}+\frac{2 j \pi}{\omega_{+}}, j=0,1,2, \cdots
$$

By Theorem 7, we have the following theorem (Ruan [74]).

Theorem 16. Suppose that $f$ and $g$ satisfy the assumptions $\left(A_{1}\right)$ and $\left(A_{2}^{\prime}\right)$. Let $\omega_{+}$and $\tau_{j}^{+}$be defined by (3.13) and (3.14), respectively.

(i) If $a d+b c>0$, then the positive equilibrium $\left(x^{*}, y^{*}\right)$ of the delayed predator-prey system (3.10) is absolutely stable.

(ii) If $a d+b c<0$, then $\left(x^{*}, y^{*}\right)$ of system (3.10) is conditionally stable: it is asymptotically stable when $\tau \in\left[0, \tau_{0}^{+}\right)$and unstable when $\tau>\tau_{0}^{+}$. Moreover, system (3.10) undergoes Hopf bifurcations at $\left(x^{*}, y^{*}\right)$ when $\tau=\tau_{j}^{+}(j=0,1,2, \cdots)$.

Remark 17. The above theorem can be used to study stability and bifurcation in some other delayed predator-prey models which are in the form of system (3.10), such as the delayed predatorprey model with mutual interference analyzed in Cao and Freedman [19] and Freedman and Rao [38], the generalized Gause-type predator-prey model with delay studied by Zhao et al. [94], the delayed predator-prey models with ratio-dependent functional response in Arditi et al. [2], Beretta and Kuang [8] and Xiao and Li [90], the delayed predator-prey models with Beddington-DeAngelis functional response in Liu and Yuan [59], etc. 
Example 18. As an example, consider the delayed Lotka-Volterra type predator-prey model:

$$
\begin{aligned}
& \dot{x}(t)=x(t)\left[r_{1}-a_{11} x(t)-a_{12} y(t)\right], \\
& \dot{y}(t)=y(t)\left[-r_{2}+a_{21} x(t-\tau)\right],
\end{aligned}
$$

where $r_{1}, r_{2}, a_{11}, a_{12}$ and $a_{21}$ are positive constants. If (3.8) holds, then system (3.15) has a positive equilibrium $\left(x^{*}, y^{*}\right)$, where

$$
x^{*}=\frac{r_{2}}{a_{21}}, \quad y^{*}=\frac{r_{1} a_{21}-r_{2} a_{11}}{a_{12} a_{21}} .
$$

Since $d=0$, we have $a d+b c=-a_{12} a_{21}<0$ and

$$
p=a_{11} x^{*}>0, r=0, s=0, q=a_{12} a_{21} x^{*} y^{*}>0 .
$$

Thus,

$$
\omega_{+}=\left\{-\frac{1}{2}\left(a_{11} x^{*}\right)^{2}+\left[\frac{1}{4}\left(\left(a_{11} x^{*}\right)^{4}+\left(a_{12} a_{21} x^{*} y^{*}\right)^{2}\right]^{\frac{1}{2}}\right\}^{\frac{1}{2}}\right.
$$

and

$$
\tau_{j}^{+}=\frac{1}{\omega_{+}} \arccos \left\{\frac{\omega_{+}^{2}}{a_{12} a_{21} x^{*} y^{*}}\right\}+\frac{2 j \pi}{\omega_{+}}, j=0,1,2, \cdots
$$

Corollary 19. Suppose (3.8) holds. Then the positive equilibrium $\left(x^{*}, y^{*}\right)$ of system (3.15) is conditionally stable: it is asymptotically stable when $\tau \in\left[0, \tau_{0}^{+}\right)$and unstable when $\tau>\tau_{0}^{+}$, where $\tau_{0}^{+}$is defined by (3.17). Moreover, when $\tau=\tau_{0}^{+}$Hopf bifurcation occurs at $\left(x^{*}, y^{*}\right)$.

\subsection{Delayed Prey Specific Growth}

Now we consider a Kolmogorov-type predator-prey model with a discrete delay appearing in the intra-specific interaction term of the prey equation:

$$
\begin{aligned}
& \dot{x}(t)=x(t) f(x(t-\tau), y(t)), \\
& \dot{y}(t)=y(t) g(x(t), y(t)),
\end{aligned}
$$

where $\tau \geq 0$ is a constant, $C=C([-\tau, 0], R), f: C \times R \rightarrow R$ and $g: R^{2} \rightarrow R$ satisfy the assumptions $\left(A_{1}\right)$ and $\left(A_{2}\right)$. The initial values of system (3.18) are

$$
x(\theta)=\phi(\theta) \geq 0, \quad \theta \in[-\tau, 0], \quad y(0)=y_{0} \geq 0 .
$$

System (3.18) has the same positive equilibrium $\left(x^{*}, y^{*}\right)$ as system (3.1). Using the change of variables (3.3), the linearized system of (3.18) at $\left(x^{*}, y^{*}\right)$ is

$$
\begin{aligned}
& \dot{X}(t)=\left(a x^{*}\right) X(t-\tau)+\left(b x^{*}\right) Y(t), \\
& \dot{Y}(t)=\left(c y^{*}\right) X(t)+\left(d y^{*}\right) Y(t),
\end{aligned}
$$


where $a<0, b<0, c>0$ and $d<0$ are defined by (3.5). The characteristic equation of the linear system (3.19) has the form:

$$
\lambda^{2}-d y^{*} \lambda-b c x^{*} y^{*}+\left(-a x^{*} \lambda+a d x^{*} y^{*}\right) e^{-\lambda \tau}=0 .
$$

It is of the form of equation (2.6) with

$$
p=-d y^{*}, r=-b c x^{*} y^{*}, s=-a x^{*}, q=a d x^{*} y^{*} .
$$

Clearly, $\left(H_{1}\right)$ and $\left(H_{2}\right)$ hold. If we assume that

$$
s^{2}-p^{2}+2 r=\left(a x^{*}\right)^{2}-\left(d y^{*}\right)^{2}+2 b c x^{*} y^{*}<0
$$

and

$$
a d+b c<0
$$

then

$$
r^{2}-q^{2}=-(a d+b c)(a d-b c)\left(x^{*} y^{*}\right)^{2}>0
$$

by assumption $\left(A_{2}\right)$, that is, $\left(H_{3}\right)$ is satisfied. Therefore, all roots of characteristic equation (3.20) have negative real parts (Ruan [74]).

Theorem 20. Suppose that $f$ and $g$ satisfy the assumptions $\left(A_{1}\right)$ and $\left(A_{2}\right)$. If conditions $(3.21)$ and (3.22) are satisfied, then the positive equilibrium $\left(x^{*}, y^{*}\right)$ of system (3.18) is absolutely stable.

If we assume that

$$
a d+b c>0,
$$

then

$$
r^{2}-q^{2}=-(a d+b c)(a d-b c)\left(x^{*} y^{*}\right)^{2}<0
$$

and $\left(H_{4}\right)$ is satisfied. It follows that the characteristic equation (3.20) has a pair of purely imaginary roots $\pm i \omega_{+}$, where

$$
\begin{aligned}
& \omega_{+}=\left\{\frac { 1 } { 2 } \left[\left(a x^{*}\right)^{2}-\left(d y^{*}\right)^{2}-2 b c x^{*} y^{*}\right.\right. \\
&\left.\left.+\left(\left(\left(a x^{*}\right)^{2}-\left(d y^{*}\right)^{2}-2 b c x^{*} y^{*}\right)^{2}-4\left(b^{2} c^{2}-a^{2} d^{2}\right)\left(x^{*} y^{*}\right)^{2}\right)^{\frac{1}{2}}\right]\right\}^{\frac{1}{2}} .
\end{aligned}
$$

Denote

$$
\tau_{j}^{+}=\frac{1}{\omega_{+}} \arccos \left\{\frac{b c d\left(y^{*}\right)^{2}}{a\left[\omega_{+}^{2}+\left(d y^{*}\right)^{2}\right]}\right\}+\frac{2 j \pi}{\omega_{+}}, j=0,1,2, \cdots
$$

By Theorem 7, we have the following result on the stability of the positive equilibrium $\left(x^{*}, y^{*}\right)$ of system (3.18) (Ruan [74]).

Theorem 21. Suppose that $f$ and $g$ satisfy the assumptions $\left(A_{1}\right)$ and $\left(A_{2}\right)$ and condition (3.23) holds. Let $\omega_{+}$and $\tau_{j}^{+}(j=0,1,2, \cdots)$ be defined by (3.24) and (3.25), respectively. The the positive steady state $\left(x^{*}, y^{*}\right)$ of system (3.18) is conditionally stable. More precisely, we have 
(i) if $\tau \in\left[0, \tau_{0}^{+}\right)$, then $\left(x^{*}, y^{*}\right)$ is asymptotically stable;

(ii) if $\tau>\tau_{0}^{+}$, then $\left(x^{*}, y^{*}\right)$ is unstable;

(iii) if $\tau=\tau_{j}^{+}(j=0,1,2, \cdots)$, then system (3.18) undergoes Hopf bifurcations at $\left(x^{*}, y^{*}\right)$.

Finally, if we assume that condition (3.22),

$$
s^{2}-p^{2}+2 r=\left(a x^{*}\right)^{2}-\left(d y^{*}\right)^{2}+2 b c x^{*} y^{*}>0
$$

and

$$
\begin{aligned}
& \left(s^{2}+s r-p^{2}\right)^{2}-4\left(r^{2}-q^{2}\right) \\
& \quad=\left[\left(a x^{*}\right)^{2}-\left(d y^{*}\right)^{2}+2 b c x^{*} y^{*}\right]^{2}+(a d+b c)(a d-b c)\left(x^{*} y^{*}\right)^{2}>0
\end{aligned}
$$

hold, then the characteristic equation (3.20) has two pairs of purely imaginary roots $\pm i \omega_{ \pm}$, where $\omega_{+}$is given in (3.24) and $\omega_{-}$is defined as follows

$$
\begin{aligned}
\omega_{-}=\{ & \frac{1}{2}\left[\left(a x^{*}\right)^{2}-\left(d y^{*}\right)^{2}-2 b c x^{*} y^{*}\right. \\
& \left.\left.-\left(\left(\left(a x^{*}\right)^{2}-\left(d y^{*}\right)^{2}-2 b c x^{*} y^{*}\right)^{2}-4\left(b^{2} c^{2}-a^{2} d^{2}\right)\left(x^{*} y^{*}\right)^{2}\right)^{\frac{1}{2}}\right]\right\}^{\frac{1}{2}} .
\end{aligned}
$$

Denote

$$
\tau_{j}^{-}=\frac{1}{\omega_{-}} \arccos \left\{\frac{b c d\left(y^{*}\right)^{2}}{a\left[\omega_{-}^{2}+\left(d y^{*}\right)^{2}\right]}\right\}+\frac{2 j \pi}{\omega_{-}}, j=0,1,2, \cdots
$$

Again by Theorem 7, we have the following theorem on the switch of stability in system (3.18) (Ruan [74]).

Theorem 22. Suppose that $f$ and $g$ satisfy the assumptions $\left(A_{1}\right)$ and $\left(A_{2}\right)$ and conditions (3.22), (3.26) and (3.27) hold. Let $\omega_{+}$and $\tau_{j}^{+}(j=0,1,2, \cdots)$ be defined by (3.24) and (3.25), respectively and $\omega_{-}$and $\tau_{j}^{-}(j=0,1,2, \cdots)$ be defined by (3.28) and (3.29), respectively. Then there is a positive integer $k$ such that when $\tau \in\left[0, \tau_{0}^{+}\right),\left(\tau_{0}^{-}, \tau_{1}^{+}\right), \cdots,\left(\tau_{k-1}^{-}, \tau_{k}^{+}\right)$, the positive equilibrium $\left(x^{*}, y^{*}\right)$ of system (3.18) is asymptotically stable; when $\tau \in\left[0, \tau_{0}^{+}\right),\left(\tau_{0}^{-}, \tau_{1}^{+}\right), \cdots,\left(\tau_{k-1}^{-}, \tau_{k}^{+}\right)$and $\tau>\tau_{k}^{+},\left(x^{*}, y^{*}\right)$ is unstable.

Remark 23. We would like to mention that switching of stabilities in delayed predator-prey models has been observed and studied in Cushing [25] and Cushing and Saleem [26].

Example 24. As an example, consider the delayed Lotka-Volterra predator-prey model proposed by May [64]:

$$
\begin{aligned}
& \dot{x}(t)=x(t)\left[r_{1}-a_{11} x(t-\tau)-a_{12} y(t)\right], \\
& \dot{y}(t)=y(t)\left[-r_{2}+a_{21} x(t)-a_{22} y(t)\right],
\end{aligned}
$$


where $r_{i}, a_{i j}(i, j=1,2)$ are positive constants. If (3.8) holds, then system (3.30) has a positive equilibrium $\left(x^{*}, y^{*}\right)$ given by (3.9). Condition $a d+b c>0$ again becomes $a_{11} a_{22}-a_{12} a_{21}>0$. Also,

$$
\begin{aligned}
\omega_{ \pm}=\{ & \frac{1}{2}\left[\left(a_{11} x^{*}\right)^{2}-\left(a_{22} y^{*}\right)^{2}-2 a_{12} a_{21} x^{*} y^{*}\right. \\
& \left.\left.\left. \pm\left[\left(a_{11} x^{*}\right)^{2}+\left(a_{22} y^{*}\right)^{2}\right)^{2}-4\left(\left(a_{11} x^{*}\right)^{2}-\left(a_{22} y^{*}\right)^{2}\right) a_{12} a_{21} x^{*} y^{*}\right]\right]^{\frac{1}{2}}\right\}^{\frac{1}{2}}
\end{aligned}
$$

and

$$
\tau_{j}^{ \pm}=\frac{1}{\omega_{ \pm}} \arccos \left\{\frac{-a_{22}\left(y^{*}\right)^{2}}{a_{11}\left[\omega_{ \pm}^{2}+\left(a_{22} y^{*}\right)^{2}\right]}\right\}+\frac{2 j \pi}{\omega_{ \pm}}, \quad j=0,1,2, \cdots
$$

We have the following result on the stability of system (3.30).

Corollary 25. Let $\omega_{ \pm}$and $\tau_{j}^{ \pm}$be defined by (3.31) and (3.32), respectively.

(i) If $\left(a_{11} x^{*}\right)^{2}-\left(a_{22} y^{*}\right)^{2}+2 a_{12} a_{21} x^{*} y^{*}<0$ and $a_{11} a_{22}-a_{12} a_{21}<0$, then the equilibrium $\left(x^{*}, y^{*}\right)$ of system (3.30) is absolutely stable.

(ii) If $a_{11} a_{22}-a_{12} a_{21}>0$, then $\left(x^{*}, y^{*}\right)$ is conditionally stable: it is asymptotically stable when $\tau \in\left[0, \tau_{0}^{+}\right)$and unstable when $\tau>\tau_{0}^{+}$. A Hopf bifurcation occurs when $\tau=\tau_{0}^{+}$.

(iii) If $\left(a_{11} x^{*}\right)^{2}-\left(a_{22} y^{*}\right)^{2}+2 a_{12} a_{21} x^{*} y^{*}>0$ and $a_{11} a_{22}-a_{12} a_{21}<0$, then there is a positive integer $k$, such that the equilibrium $\left(x^{*}, y^{*}\right)$ switches $k$ times from stability to instability to stability; that is, $\left(x^{*}, y^{*}\right)$ is asymptotically stable when

$$
\tau \in\left[0, \tau_{0}^{+}\right),\left(\tau_{0}^{-}, \tau_{1}^{+}\right), \cdots,\left(\tau_{k-1}^{-}, \tau_{k}^{+}\right)
$$

and unstable when

$$
\tau \in\left[\tau_{0}^{+}, \tau_{0}^{-}\right),\left[\tau_{1}^{+}, \tau_{1}^{-}\right), \cdots,\left[\tau_{k-1}^{+}, \tau_{k-1}^{-}\right) \text {and } \tau>\tau_{k}^{+}
$$

Using a global Hopf bifurcation result due to Wu [88], Song and Wei [80] showed that for the delayed Lotka-Volterra predator-prey model (3.30) local Hopf bifurcation implies global Hopf bifurcation after the second critical delay value. They considered a numerical example

$$
\begin{aligned}
& \dot{x}(t)=x(t)[1-x(t-\tau)-y(t)], \\
& \dot{y}(t)=y(t)[-1+3 x(t)-y(t)],
\end{aligned}
$$

which has a positive equilibrium $E^{*}=(0.5,0.5)$. One can find that $\tau_{0}^{+}=2.2143, \tau_{0}^{-}=4.4288, \tau_{1}^{+}$ $=8.4975, \tau_{1}^{-}=13.3286, \tau_{2}^{+}=14.7807, \tau_{2}^{-}=22.2142, \tau_{3}^{+}=21.0639, \ldots$ Notice that $\tau_{3}^{+}<$ $\tau_{2}^{-}$. Thus, the positive equilibrium $E^{*}=(0.5,0.5)$ switches stability 2 times, which is stable for $\tau \in(0,2.2143),(4.4288,8.4975),(13.3286,14.7807)$, unstable for $\tau \in[2.2143,4.4288),[8.4975$, 13.3286) and for $\tau \geq \tau_{2}^{+}=14.7807$. They also presented some numerical simulations to show the stability switch. 


\section{A Delayed Predator-Prey System with Nonmonotonic Func- tional Response}

In this section, we consider the delayed predator-prey system with nonmonotone functional response (1.13) and investigate the effect of the time delay on bifurcations of the system. When $\tau=0$, there exist some parameter values such that system (1.13) has a positive equilibrium which is a stable multiple focus in the interior of the first quadrant and there are some other parameter values of $r, K, a, \mu$, and $D$ such that system (1.13) exhibits Bogdanov-Takens bifurcation (see Ruan and Xiao [77]). Xiao and Ruan [92] showed that when $\tau>0$ this equilibrium becomes unstable and Hopf bifurcation always occurs for some $\tau_{k}>0$. Moreover, the delayed system (1.13) still has a Bogdanov-Takens singularity for any $\tau>0$. We now introduce the results from Xiao and Ruan [92].

\subsection{Hopf Bifurcation}

It has been shown in [77] that in the model (1.13) with $\tau=0$, when $\mu^{2}>4 a D^{2}$ and $K>$ $\frac{\mu+\sqrt{\mu^{2}-4 a D^{2}}}{2 D}$, there are two positive equilibria: a focus $\left(x_{1}, y_{1}\right)$ and a hyperbolic saddle $\left(x_{2}, y_{2}\right)$. Moreover, when $K=\frac{2 \mu-\sqrt{\mu^{2}-4 a D^{2}}}{2 D}$ the focus $\left(x_{1}, y_{1}\right)$ is a multiple focus, at which the third focal value (i.e. the Liapunov number) $\alpha_{3}$ is equal to zero if and only if $\mu=\frac{18+2 \sqrt{6}}{3} a D^{2}$. Thus, $\left(x_{1}, y_{1}\right)$ can be a multiple focus of multiplicity at least two for some parameter values. Notice that when $4 a D^{2}<\mu<\frac{18+2 \sqrt{6}}{3} a D^{2}$ and $K=\frac{2 \mu-\sqrt{\mu^{2}-4 a D^{2}}}{2 D},\left(x_{1}, y_{1}\right)$ is a multiple focus of multiplicity one, which is stable. We will consider the effect of the delay on the stability of $\left(x_{1}, y_{1}\right)$ when it is a stable multiple focus of multiplicity one as $\tau=0$ and will show that a small delay can change the stability of the equilibrium. Choosing $\tau$ as the bifurcation parameter, we will discuss the Hopf bifurcation of system (1.13) for a class of parameters $a, \mu, K$, and $D$ by using the normal form theory developed by Faria and Magalhães [32, 34].

The following lemma ensures that $\left(x_{1}, y_{1}\right)$ is a stable focus of multiplicity one and there are no nontrivial closed orbits (periodic orbits or homoclinic orbits) when $\tau=0$ (Xiao and Ruan [92]).

Lemma 26. If $4 a D^{2}<\mu^{2} \leq \frac{16}{3} a D^{2}$ and $K=\frac{2 \mu-\sqrt{\mu^{2}-4 a D^{2}}}{2 D}$, then system (1.13) with $\tau=0$ has an interior equilibrium $\left(x_{1}, y_{1}\right)$, which is stable, and there are no nontrivial closed orbits (periodic orbits or homoclinic orbits) in the interior of the first quadrant, where

$$
x_{1}=\frac{\mu-\sqrt{\mu^{2}-4 a D^{2}}}{2 D}, \quad y_{1}=r\left(1-\frac{x_{1}}{K}\right)\left(a+x_{1}^{2}\right) .
$$

In fact, the equilibrium $\left(x_{1}, y_{1}\right)$ is a focus of multiplicity one for small positive $\tau$, which becomes unstable (Xiao and Ruan [92]).

Theorem 27. Suppose that $4 a D^{2}<\mu^{2} \leq \frac{16}{3} a D^{2}$ and $K=\frac{2 \mu-\sqrt{\mu^{2}-4 a D^{2}}}{2 D}$. Then system (1.13) has an interior equilibrium $\left(x_{1}, y_{1}\right)$ which is unstable for $0<\tau \ll 1$. 
Proof. Let $X_{1}=x-x_{1}, X_{2}=y-y_{1}$. Then system (1.13) becomes

$$
\begin{aligned}
& \dot{X}_{1}(t)=-\frac{x_{1}}{a+x_{1}^{2}} X_{2}(t)+\sum_{i+j \geq 2} \frac{1}{i ! j !} h_{i j}^{(1)} X_{1}^{i}(t) X_{2}^{j}(t), \\
& \dot{X}_{2}(t)=\frac{\mu y_{1}\left(a-x_{1}^{2}\right)}{\left(a+x_{1}^{2}\right)^{2}} X_{1}(t-\tau)+\sum_{i+j \geq 2} \frac{1}{i ! j !} h_{i j}^{(2)} X_{1}^{i}(t-\tau) X_{2}^{j}(t),
\end{aligned}
$$

where $i, j \geq 0$,

$$
\begin{gathered}
h_{i j}^{(1)}=\left.\frac{\partial^{i+j} h^{(1)}}{\partial^{i} x \partial^{j} y}\right|_{\left(x_{1}, y_{1}\right)}, \quad h_{i j}^{(2)}=\left.\frac{\partial^{i+j} h^{(2)}}{\partial^{i} x \partial^{j} y}\right|_{\left(x_{1}, y_{1}\right)}, \\
h^{(1)}=r x\left(1-\frac{x}{K}\right)-\frac{x y}{a+x^{2}}, \quad h^{(2)}=y\left(\frac{\mu x}{a+x^{2}}-D\right) .
\end{gathered}
$$

To study the stability of the origin, consider the linearized system at $(0,0)$

$$
\begin{aligned}
& \dot{X}_{1}(t)=-\frac{x_{1}}{a+x_{1}^{2}} X_{2}(t) \\
& \dot{X}_{2}(t)=\frac{\mu y_{1}\left(a-x_{1}^{2}\right)}{\left(a+x_{1}^{2}\right)^{2}} X_{1}(t-\tau) .
\end{aligned}
$$

System (4.2) has the characteristic equation

$$
\Delta(\lambda, \tau)=\lambda^{2}+q e^{-\lambda \tau},
$$

where

$$
q=\frac{\mu x_{1} y_{1}\left(a-x_{1}^{2}\right)}{\left(a+x_{1}^{2}\right)^{3}}>0 .
$$

It is clear that the characteristic equation (4.3) has no real roots and $\Delta(\lambda, 0)=0$ has only a pair of conjugate purely imaginary roots $\pm i \sqrt{q}$.

Assume that $\lambda=u+i v$ is a root of (4.3) for $\tau>0$. Then we have

$$
\begin{aligned}
& H_{1}(u, v, \tau)=u^{2}-v^{2}+q e^{-u \tau} \cos v \tau=0 \\
& H_{2}(u, v, \tau)=2 u v-q e^{-u \tau} \sin v \tau=0 .
\end{aligned}
$$

The Implicit Function Theorem implies that $\left(H_{1}(u, v, \tau), H_{2}(u, v, \tau)\right)=(0,0)$ defines $u, v$ as functions of $\tau$, i.e. $u=u(\tau)$ and $v=v(\tau)$, in a neighborhood of $\tau=0$ such that

$$
u(0)=0, \quad v(0)=\sqrt{q},\left.\quad \frac{d}{d \tau} u(\tau)\right|_{\tau=0}>0 .
$$

Therefore, $u(\tau)>0$ as $\tau>0$. This completes the proof of the theorem. 
Thus, there exist

$$
\tau_{k}=\frac{2 k \pi}{\sqrt{q}}, \quad k=0,1,2, \ldots
$$

such that the characteristic equation (4.3) has two simple complex roots $u(\tau) \pm i v(\tau)$ which cross the imaginary axis transversely at $\tau=\tau_{k}$ :

$$
u\left(\tau_{k}\right)=0, \quad v\left(\tau_{k}\right)=\sqrt{q}>0, \quad u^{\prime}\left(\tau_{k}\right)>0,
$$

and (4.3) has no other roots when $\tau=\tau_{k}$ in the imaginary axis which are multiples of $i \sqrt{q}$. Hence, Hopf bifurcation may occur at $\tau=\tau_{k}$.

Next, choosing $\tau$ as a bifurcation parameter and following the normal form theory developed by Faria and Magalhães [34], we discuss the explicit expressions of the normal form of system (4.1) in terms of the original parameters in a small neighborhood of $\tau_{k}$. For $\tau>0$, rewrite system (4.1) as follows:

$$
\begin{aligned}
& \dot{X}_{1}(t)=\tau\left[-\frac{x_{1}}{a+x_{1}^{2}} X_{2}(t)+\sum_{i+j \geq 2} \frac{1}{i ! j !} h_{i j}^{(1)} X_{1}^{i}(t) X_{2}^{j}(t)\right] \\
& \dot{X}_{2}(t)=\tau\left[\frac{\mu y_{1}\left(a-x_{1}^{2}\right)}{\left(a+x_{1}^{2}\right)^{2}} X_{1}(t-1)+\sum_{i+j \geq 2} \frac{1}{i ! j !} h_{i j}^{(2)} X_{1}^{i}(t-1) X_{2}^{j}(t)\right],
\end{aligned}
$$

and the linearized system is

$$
\begin{aligned}
& \dot{X}_{1}(t)=-\frac{\tau x_{1}}{a+x_{1}^{2}} X_{2}(t), \\
& \dot{X}_{2}(t)=\frac{\tau \mu y_{1}\left(a-x_{1}^{2}\right)}{\left(a+x_{1}^{2}\right)^{2}} X_{1}(t-1) .
\end{aligned}
$$

Let $A$ be the generator of the linear semigroup corresponding to (4.5). When $\tau=\tau_{k}, A$ has a pair of purely imaginary characteristic roots $\pm i 2 k \pi$, which are simple, and no other characteristic roots with zero real part. Define $\nu=\tau-\tau_{k}$. System (4.4) can be written as

$$
\begin{aligned}
\dot{X}_{1}(t)= & -\frac{\tau_{k} x_{1}}{a+x_{1}^{2}} X_{2}(t)-\frac{\nu x_{1}}{a+x_{1}^{2}} X_{2}(t)+\left(\tau_{k}+\nu\right) \sum_{i+j \geq 2} \frac{1}{i ! j !} h_{i j}^{(1)} X_{1}^{i}(t) X_{2}^{j}(t), \\
\dot{X}_{2}(t)= & \frac{\tau_{k} \mu y_{1}\left(a-x_{1}^{2}\right)}{\left(a+x_{1}^{2}\right)^{2}} X_{1}(t-1)+\frac{\nu \mu y_{1}\left(a-x_{1}^{2}\right)}{\left(a+x_{1}^{2}\right)^{2}} X_{1}(t-1) \\
& +\left(\tau_{k}+\nu\right) \sum_{i+j \geq 2} \frac{1}{i ! j !} h_{i j}^{(2)} X_{1}^{i}(t-1) X_{2}^{j}(t)
\end{aligned}
$$

or in the vector form

$$
\dot{X}(t)=\left(\begin{array}{cc}
0 & -\frac{\tau_{k} x_{1}}{a+x_{1}^{2}} \\
\frac{\tau_{k} \mu y_{1}\left(a-x_{1}^{2}\right)}{\left(a+x_{1}^{2}\right)^{2}} & 0
\end{array}\right)\left(\begin{array}{c}
X_{1}(t-1) \\
X_{2}(t)
\end{array}\right)+H_{0}\left(X_{t}, \nu\right) .
$$


For any $\nu$, system (4.6) has an equilibrium at $(0,0)$. The phase space is $C_{1}=C\left([-1,0] ; R^{2}\right)$. Fix a $k \in N=\{1,2, \ldots\}$ and define $\Lambda=\{-i 2 k \pi, i 2 k \pi\}$. We apply the normal form theory in [34] to system (4.6).

Let the phase space $C_{1}$ be decomposed by $\Lambda$ as $C_{1}=P \oplus Q$, where $P$ is the generalized eigenspace associated with $\Lambda$. Consider the bilinear form $(\cdot, \cdot)$ associated with the linear system

$$
\begin{aligned}
& \dot{X}_{1}(t)=-\frac{\tau_{k} x_{1}}{a+x_{1}^{2}} X_{2}(t), \\
& \dot{X}_{2}(t)=\frac{\tau_{k} \mu y_{1}\left(a-x_{1}^{2}\right)}{\left(a+x_{1}^{2}\right)^{2}} X_{1}(t-1) .
\end{aligned}
$$

Let $\Phi$ and $\Psi$ be bases for $P$ and $P^{*}$ associated with the eigenvalues $\pm i 2 k \pi$ of the adjoint equations, respectively. Normalize them so that $(\Phi, \Psi)=I$. Here, it is convenient to combine one complex coordinate and two complex conjugate basis vectors to describe a two-dimensional real subspace $P$. Consider system (4.6) in $C([-1,0] ; \mathbb{C})$, still denoted by $C_{1}$.

Note that $\dot{\Phi}=\Phi B$, where $B=\operatorname{diag}(i 2 k \pi,-i 2 k \pi)$ is a diagonal matrix. Therefore, $\Phi$ and $\Psi$ are $2 \times 2$ matrices of the form

$$
\begin{gathered}
\Phi(\theta)=\left[\phi_{1}(\theta), \phi_{2}(\theta)\right], \quad \phi_{1}(\theta)=e^{i 2 k \pi \theta} v, \quad \phi_{2}(\theta)=\overline{\phi_{1}(\theta)}, \quad-1 \leq \theta \leq 0, \\
\Psi(s)=\left(\begin{array}{c}
\psi_{1}(s) \\
\psi_{2}(s)
\end{array}\right), \quad \psi_{1}(s)=e^{-i 2 k \pi s} u^{T}, \quad \psi_{2}(s)=\overline{\psi_{1}(s)}, \quad 0 \leq s \leq 1,
\end{gathered}
$$

where the bar means complex conjugation, $u^{T}$ is the transpose of $u$, and $u, v$ are vectors in $\mathbb{C}^{2}$,

$$
u=\left(\begin{array}{l}
u_{1} \\
u_{2}
\end{array}\right)=\left(\begin{array}{c}
\frac{1}{2+i 2 k \pi} \\
\frac{i x}{\sqrt{q}\left(a+x_{1}^{2}\right)(2+i 2 k \pi)}
\end{array}\right), \quad v=\left(\begin{array}{c}
v_{1} \\
v_{2}
\end{array}\right)=\left(\begin{array}{c}
1 \\
\frac{-i \sqrt{q}\left(a+x_{1}^{2}\right)}{x_{1}}
\end{array}\right) .
$$

Enlarging the phase space $C_{1}$ by considering the space $B C$ and using the decomposition $X_{t}=$ $\Phi z(t)+y_{t}, z \in \mathbb{C}^{2}, y_{t} \in Q^{\prime}$, we decompose system (4.6) as follows

$$
\begin{aligned}
& \dot{z}=B z+\Psi(0) H_{0}(\Phi z+y, \nu), \\
& \dot{y}=A_{Q^{\prime}} y+(I-\pi) X_{0} H_{0}(\Phi z+y, \nu) .
\end{aligned}
$$

Following the procedure of reducing normal forms in [34], we consider

$$
\Psi(0) H_{0}(\Phi z+y, \nu)=\frac{1}{2} h_{2}(z, y, \nu)+\frac{1}{3 !} h_{3}(z, y, \nu)+\text { h.o.t. },
$$

where $h_{j}(z, y, \nu)(j=1,2)$ are homogeneous polynomials in $(z, y, \nu)$ of degree $j$ with coefficients in $\mathbb{C}^{2}$ and h.o.t. stands for higher order terms. Thus, in a finite dimensional locally invariant manifold tangent to the invariant subspace $P$ of (4.7) at $x=0, \nu=0$, the normal form of (4.8) is given by

$$
\dot{z}=B z+\frac{1}{2} \bar{h}_{2}(z, 0, \nu)+\frac{1}{3 !} \bar{h}_{3}(z, 0, \nu)+\text { h.o.t. }
$$


where $\bar{h}_{2}, \bar{h}_{3}$ are the second and third order terms in $(z, \nu)$, respectively. Using the notation in [34], we have

$$
\bar{h}_{2}(z, 0, \nu)=\operatorname{Proj}_{\operatorname{Ker}\left(M_{2}^{\prime}\right)} h_{2}(z, 0, \nu)
$$

where

$$
\begin{gathered}
\operatorname{Ker}\left(M_{2}^{\prime}\right)=\operatorname{span}\left\{\left(\begin{array}{c}
z_{1} \nu \\
0
\end{array}\right),\left(\begin{array}{c}
0 \\
z_{2} \nu
\end{array}\right)\right\}, \\
h_{2}(z, 0, \nu)=\left(\begin{array}{c}
2 i \sqrt{q} u^{T} v z_{1} \nu+a_{20} z_{1}^{2}+a_{11} z_{1} z_{2}+a_{02} z_{2}^{2} \\
-2 i \sqrt{q} \bar{u}^{T} \bar{v} z_{2} \nu+\bar{a}_{20} z_{1}^{2}+\bar{a}_{11} z_{1} z_{2}+\bar{a}_{02} z_{2}^{2}
\end{array}\right),
\end{gathered}
$$

in which

$$
\begin{aligned}
& a_{20}=\tau_{k}\left(h_{20}^{(1)} u_{1}+h_{11}^{(1)} v_{2} u_{1}+h_{20}^{(2)} e^{-4 i k \pi} u_{2}+h_{11}^{(2)} e^{-2 i k \pi} v_{2} u_{2}\right), \\
& a_{11}=\tau_{k}\left(2 h_{20}^{(1)} u_{1}+2 h_{20}^{(2)} u_{2}+h_{11}^{(2)}\left(e^{-2 i k \pi} \bar{v}_{2}+e^{2 i k \pi} v_{2}\right) u_{2}\right), \\
& a_{02}=\tau_{k}\left(h_{20}^{(1)} u_{1}+h_{11}^{(1)} \bar{v}_{2} u_{1}+h_{20}^{(2)} e^{4 i k \pi} u_{2}+h_{11}^{(2)} e^{2 i k \pi} \bar{v}_{2} u_{2}\right) .
\end{aligned}
$$

Therefore,

$$
\frac{1}{2} \bar{h}_{2}(z, 0, \nu)=\left(\begin{array}{c}
i \sqrt{q} u^{T} v z_{1} \nu \\
-i \sqrt{q} \bar{u}^{T} \bar{v} z_{2} \nu
\end{array}\right)
$$

To eliminate these nonresonant terms in the quadratic terms $h_{2}(z, 0, \nu)$, we have to make a series of transformations of variables, which can change the coefficients of the cubic terms of $h_{3}(z, 0, \nu)$. Notice that

$$
\operatorname{Ker}\left(M_{3}^{\prime}\right)=\operatorname{span}\left\{\left(\begin{array}{c}
z_{1}^{2} z_{2} \\
0
\end{array}\right),\left(\begin{array}{c}
z_{1} \nu \\
0
\end{array}\right),\left(\begin{array}{c}
0 \\
z_{1} z_{2}^{2}
\end{array}\right),\left(\begin{array}{c}
0 \\
z_{2} \nu^{2}
\end{array}\right)\right\} .
$$

However, the terms $O\left(|z| \nu^{2}\right)$ are irrelevant to determine the generic Hopf bifurcation. Hence, we only need to compute the coefficient of $z_{1}^{2} z_{2}$. After some computations we find that the coefficient of $z_{1}^{2} z_{2}$ is

$$
c=\frac{i}{4 k \pi}\left(a_{20} a_{11}-2\left|a_{11}\right|^{2}-\frac{1}{3}\left|a_{02}\right|^{2}\right)+\frac{1}{2} a_{21},
$$

where

$$
a_{21}=\tau_{k}\left[3 h_{30}^{(1)} u_{1}+h_{21}^{(1)} v_{2} u_{1}+3 h_{30}^{(2)} e^{-2 i k \pi} u_{2}+h_{21}^{(2)}\left(e^{-4 i k \pi} \bar{v}_{2}+2 v_{2}\right) u_{2}\right]
$$

Thus,

$$
\frac{1}{3 !} \bar{h}_{3}(z, 0, \nu)=\left(\begin{array}{c}
c z_{1}^{2} z_{2} \\
\bar{c} z_{1} z_{2}^{2}
\end{array}\right)+O\left(|z| \nu^{2}\right)
$$

The normal form (4.9) relative to $P$ can be written in real coordinates $(x, y)$, through the change of variables $z_{1}=x-i y, z_{2}=x+i y$. Followed by the use of polar coordinates $(r, \theta), x=r \cos \theta, y=$ $\sin \theta$, this normal form becomes

$$
\begin{aligned}
& \dot{r}=c_{1} \nu r+c_{2} r^{3}+O\left(\nu^{2} r+|(r, \nu)|^{4}\right), \\
& \dot{\theta}=-2 k \pi+O(|(r, \nu)|),
\end{aligned}
$$

where $c_{1}=\frac{k \pi \sqrt{q}}{1+k^{2} \pi^{2}}, c_{2}=\operatorname{Re}(c)$.

Summarizing the above analysis, we have the following theorem (Xiao and Ruan [92]). 
Theorem 28. If $c_{2} \neq 0$ and $\tau_{k}>0$, then system (4.6) exhibits a generic Hopf bifurcation. The periodic orbits of system (4.6) bifurcating from the origin and $\nu=0$ satisfy

$$
r(t, \nu)=\sqrt{-\frac{c_{1} \nu}{c_{2}}}+O(\nu), \quad \theta(t, \nu)=-2 k \pi t+O\left(|\nu|^{\frac{1}{2}}\right)
$$

so that

(i) if $c_{1} c_{2}<0\left(c_{1} c_{2}>0\right.$ respectively), there exists a unique nontrivial periodic orbit in a neighborhood of $r=0$ for $\nu>0$ ( $\nu<0$ respectively) and no nontrivial periodic orbits for $\nu<0(\nu>0$ respectively);

(ii) the nontrivial periodic solutions in the center manifold are stable if $c_{2}<0$ and unstable if $c_{2}>0$. They are always unstable in the whole phase space $C_{1}$ since the center manifold are unstable for $\tau_{k}>0$.

\subsection{Bogdanov-Takens Bifurcation}

System (1.13) with $\tau=0$ has a unique interior equilibrium $\left(x_{0}, y_{0}\right)$ if and only if $\mu^{2}-4 a D^{2}=0$ and $\mu<2 K D$. Furthermore, when $\mu=K D$ the equilibrium $\left(x_{0}, y_{0}\right)$ is a cusp of codimension 2 (i.e. a Bogdanov-Takens singularity) as shown in [77], and $x_{0}=\mu / 2 D, y_{0}=\mathrm{ra}$. Since time delay does not affect the number and location of equilibria, $\left(x_{0}, y_{0}\right)$ is still a unique interior equilibrium for the delayed system (1.13) when $\mu^{2}-4 a D^{2}=0$ and $\mu=K D$. We will show that the interior equilibrium $\left(x_{0}, y_{0}\right)$ is also a Bogdanov-Takens singularity for system (1.13). Denote by $\mu_{0}, a_{0}, D_{0}$, and $K_{0}$ if they satisfy $\mu^{2}-4 a D^{2}=0$ and $\mu=K D$.

Consider the delayed model

$$
\begin{aligned}
& \dot{x}(t)=r x(t)\left(1-\frac{x(t)}{K_{0}}\right)-\frac{x(t) y(t)}{a_{0}+x^{2}(t)}, \\
& \dot{y}(t)=y(t)\left(\frac{\mu_{0} x(t-\tau)}{a_{0}+x^{2}(t-\tau)}-D_{0}\right)
\end{aligned}
$$

in the phase space $C:=C\left([-\tau, 0] ; R^{2}\right)$, here $\tau>0$ is a constant. It is convenient to reparametrize system (4.11) so that it becomes

$$
\begin{aligned}
& \dot{x}(t)=\tau\left[r x(t)\left(1-\frac{x(t)}{K_{0}}\right)-\frac{x(t) y(t)}{a_{0}+x^{2}(t)}\right], \\
& \dot{y}(t)=\tau\left[y(t)\left(\frac{\mu_{0} x(t-1)}{a_{0}+x^{2}(t-1)}-D_{0}\right)\right] .
\end{aligned}
$$

The advantage is that we can work in a fixed phase space $C_{1}:=C\left([-1,0] ; R^{2}\right)$ when $\tau$ varies. 
First of all, we translate the equilibrium $\left(x_{0}, y_{0}\right)$ of system (4.12) to the origin. Let $x_{1}=$ $x-x_{0}, x_{2}=y-y_{0}$. Then system (4.12) becomes

$$
\begin{aligned}
& \dot{x}_{1}(t)=\tau\left[-\frac{x_{0}}{a_{0}+x_{0}^{2}} x_{2}(t)+\sum_{i+j \geq 2} \frac{1}{i ! j !} f_{i j}^{(1)} x_{1}^{i}(t) x_{2}^{j}(t)\right], \\
& \dot{x}_{2}(t)=\tau\left[\sum_{i+j \geq 2} \frac{1}{i ! j !} f_{i j}^{(2)} x_{1}^{i}(t-1) x_{2}^{j}(t)\right],
\end{aligned}
$$

where $i, j \geq 0$,

$$
\begin{gathered}
f_{i j}^{(1)}=\left.\frac{\partial^{i+j} f^{(1)}}{\partial^{i} x \partial^{j} y}\right|_{\left(x_{0}, y_{0}\right)}, \quad f_{i j}^{(2)}=\left.\frac{\partial^{i+j} f^{(2)}}{\partial^{i} x \partial^{j} y}\right|_{\left(x_{0}, y_{0}\right)}, \\
f^{(1)}=r x\left(1-\frac{x}{K_{0}}\right)-\frac{x y}{a_{0}+x^{2}}, \quad f^{(2)}=y\left(\frac{\mu_{0} x}{a_{0}+x^{2}}-D_{0}\right) .
\end{gathered}
$$

Linearization at the zero equilibrium yields

$$
\begin{aligned}
& \dot{x}_{1}(t)=-\frac{\tau x_{0}}{a_{0}+x_{0}^{2}} x_{2}(t), \\
& \dot{x}_{2}(t)=0
\end{aligned}
$$

which has $\lambda=0$ as a double characteristic value and no other characteristic values.

Now we consider the normal form of system (4.13) at the singularity $(0,0)$. For simplicity, we rewrite system (4.14) as

$$
\dot{X}(t)=L\left(X_{t}\right),
$$

here $X(t)=\left(x_{1}(t), x_{2}(t)\right), L(\phi)=L\left(\begin{array}{c}\phi_{1}(-1) \\ \phi_{2}(0)\end{array}\right)$, and $\phi=\left(\phi_{1}, \phi_{2}\right)$. According to the normal form theory developed by Faria and Magalhães [33] we know that the center manifold of system (4.14) at the origin is two dimensional and system (4.13) can be reduced to an ODE in the plane.

Let $A_{0}$ be the infinitesimal generator of the linear system (4.14). Consider $\Lambda=\{0\}$ and denote by $P$ the invariant space of $A_{0}$ associated with the eigenvalue $\lambda=0$. Using the formal adjoint theory of RFDE in [33], we know that the phase space $C_{1}$ can be decomposed by $\Lambda$ as $C_{1}=P \oplus Q$. Let $\Phi$ and $\Psi$ be the bases for $P$ and $P^{*}$, the space associated with the eigenvalue $\lambda=0$ of the adjoint equation, respectively, and be normalized so that $(\Phi, \Psi)=I$, where $(\cdot, \cdot)$ is the bilinear form defined in section 2 of [33]. We refer to [33] for the unexplained notation and definitions. $\Phi$ and $\Psi$ are $2 \times 2$ matrices of the form:

$$
\begin{gathered}
\Phi(\theta)=\left(\begin{array}{cc}
1 & \theta \\
0 & -\frac{a_{0}+x_{0}^{2}}{\tau x_{0}}
\end{array}\right)=\left(\begin{array}{cc}
1 & \theta \\
0 & -\frac{\mu_{0}}{\tau D_{0}}
\end{array}\right), \quad-1 \leq \theta \leq 0, \\
\Psi(s)=\left(\begin{array}{cc}
1 & \frac{\tau D_{0}}{\mu_{0}} s \\
0 & -\frac{\tau D_{0}}{\mu_{0}}
\end{array}\right), \quad 0 \leq s \leq 1 .
\end{gathered}
$$


The matrix $B$ satisfying $\dot{\Phi}=\Phi B$ is given by

$$
B=\left(\begin{array}{ll}
0 & 1 \\
0 & 0
\end{array}\right)
$$

Enlarging the phase space $C_{1}$ by considering the space $B C=\left\{\phi:[-1,0] \rightarrow R^{2} ; \phi\right.$ is continuous on $[-1,0)$ with a jump discontinuity at 0$\}$, we can see that the projection of $C_{1}$ upon $P$, associated with the decomposition $C_{1}=P \oplus Q$, is now replaced by $\pi: B C \rightarrow P$, which leads to the decomposition

$$
B C=P \oplus \operatorname{Ker} \pi
$$

following [33]. Now decompose $x$ in system (4.13) according to the preceding decomposition of $B C$, in the form $x=\Phi z+y$, with $z \in R^{2}$ and $y \in \operatorname{Ker} \pi \cap D\left(A_{0}\right)=Q^{\prime}$. Hence, system (4.13) in the center manifold is equivalent to the system

$$
\dot{z}=B z+\Psi(0) F(\Phi z)
$$

where

$$
F(\phi)=\left(\begin{array}{c}
\tau \sum_{i+j \geq 2} \frac{1}{i ! j !} f_{i j}^{(1)}\left[\phi_{1}(0)\right]^{i}\left[\phi_{2}(0)\right]^{j} \\
\tau \sum_{i+j \geq 2} \frac{1}{i ! j !} f_{i j}^{(2)}\left[\phi_{1}(-1)\right]^{i}\left[\phi_{2}(0)\right]^{j}
\end{array}\right),
$$

where $\phi=\left(\phi_{1}, \phi_{2}\right)$. Writing $F$ in its Taylor expansion up to the second order terms in the form $F(z)=\frac{1}{2 !} F_{2}(z)+O\left(|z|^{3}\right)$, we have

$$
\begin{aligned}
& \dot{z}_{1}=z_{2}+\frac{1}{2 !} \tau f_{20}^{(1)} z_{1}^{2}+\tau P_{1}\left(z_{1}, z_{2}\right) \\
& \dot{z}_{2}=-\frac{1}{2 !} \frac{\tau^{2} D_{0}}{\mu_{0}} f_{20}^{(2)}\left(z_{1}-z_{2}\right)^{2}+\tau P_{2}\left(z_{1}, z_{2}\right),
\end{aligned}
$$

where $f_{20}^{(1)}=-\frac{r D_{0}}{\mu_{0}}, f_{20}^{(2)}=-r D_{0}, P_{1}$ and $P_{2}$ are $C^{\infty}$ functions in $\left(z_{1}, z_{2}\right)$ at least of the third order.

In a neighborhood of the origin, we make the inverse transformation

$$
\bar{z}_{1}=z_{1}, \quad \bar{z}_{2}=z_{2}+\frac{\tau}{2} f_{20}^{(1)} z_{1}^{2}+\tau P_{1}\left(z_{1}, z_{2}\right) .
$$

After dropping the bars, system (4.16) becomes

$$
\begin{aligned}
& \dot{z}_{1}=z_{2}, \\
& \dot{z}_{2}=\alpha z_{1}^{2}+\beta z_{1} z_{2}+\gamma z_{2}^{2}+P_{3}\left(z_{1}, z_{2}\right),
\end{aligned}
$$

here

$$
\alpha=\frac{r \tau^{2} D_{0}^{2}}{2 \mu_{0}}, \quad \beta=-\frac{r \tau D_{0}+r \tau^{2} D_{0}^{2}}{\mu_{0}}, \quad \gamma=\frac{r \tau^{2} D_{0}^{2}}{2 \mu_{0}},
$$

and $P_{3}$ is a $C^{\infty}$ function in $\left(z_{1}, z_{2}\right)$ at least of the third order whose coefficients are functions of $\tau, r, D_{0}, \mu_{0}, a_{0}$, and $K_{0}$. By the nonresonance conditions among the set $\Lambda$, we can eliminate the $z_{2}^{2}$ term in the second equation of system (4.17) and obtain the following

$$
\begin{aligned}
& \dot{z}_{1}=z_{2}, \\
& \dot{z}_{2}=\alpha z_{1}^{2}+\beta z_{1} z_{2}+P_{4}\left(z_{1}, z_{2}\right),
\end{aligned}
$$


where $P_{4}$ is a $C^{\infty}$ function in $\left(z_{1}, z_{2}\right)$ at least of the third order. By the above argument, we have the following result (Xiao and Ruan [92]).

Theorem 29. For any $\tau>0$, the equilibrium $\left(x_{0}, y_{0}\right)$ of system (4.11) is a Bogdanov-Takens singularity, the dynamics in a neighborhood of $\left(x_{0}, y_{0}\right)$ is generically determined by the quadratic terms of system (4.11).

Now we determine a versal unfolding for the original system (4.11) or system (4.12) with a Bogdanov-Takens singularity, i.e., to determine which of the parameters $r, K, D, a, \mu$, and $\tau$ can be chosen as bifurcation parameters such that system (4.11) exhibits Bogdanov-Takens bifurcation. We cannot get any versal unfoldings of this Bogdanov-Takens singularity if we require that system (4.12) always has an equilibrium $\left(x_{0}, y_{0}\right)$, as stated in [33], for all bifurcation parameters. However, if we give up this restraint and assume the following condition instead

(H) System (4.12) has a Bogdanov-Takens singularity $\left(x_{0}, y_{0}\right)$ when all bifurcation parameters equal to zero,

then we can obtain a versal unfolding of this Bogdanov-Takens singularity depending on all parameters of the original system. For this purpose, choose $K$ and $D$ in system (4.12) as the bifurcation parameters, i.e. consider $\frac{1}{K_{0}}+\lambda_{1}$ and $D_{0}+\lambda_{2}$, where $\lambda_{1}$ and $\lambda_{2}$ vary in a small neighborhood of $(0,0)$. Adding these perturbations to system (4.12), we obtain

$$
\begin{aligned}
& \dot{x}(t)=\tau\left[r x(t)\left(1-\frac{x(t)}{K_{0}}\right)-\frac{x(t) y(t)}{a_{0}+x^{2}(t)}-r \lambda_{1} x^{2}(t)\right], \\
& \dot{y}(t)=\tau y(t)\left(\frac{\mu_{0} x(t-1)}{a_{0}+x^{2}(t-1)}-D_{0}-\lambda_{2}\right) .
\end{aligned}
$$

When $\lambda_{1}=\lambda_{2}=0$, system (4.19) has a Bogdanov-Takens singularity $\left(x_{0}, y_{0}\right)$ and there exists a two-dimensional center manifold.

Let $y_{1}=x-x_{0}, y_{2}=y-y_{0}$. Then system (4.19) becomes

$$
\begin{aligned}
& \dot{y}_{1}(t)=-r \tau x_{0}^{2} \lambda_{1}-2 r \tau x_{0} \lambda_{1} y_{1}(t)-\frac{\tau x_{0}}{a_{0}+x_{0}^{2}} y_{2}(t)+\sum_{i+j \geq 2} \frac{1}{i ! j !} \tau g_{i j}^{(1)} y_{1}^{i}(t) y_{2}^{j}(t), \\
& \dot{y}_{2}(t)=-\tau y_{0} \lambda_{2}-\tau \lambda_{2} y_{2}(t)+\sum_{i+j \geq 2} \frac{1}{i ! j !} \tau g_{i j}^{(2)} y_{1}^{i}(t-1) y_{2}^{j}(t)
\end{aligned}
$$

where $i, j \geq 0$,

$$
\begin{gathered}
g_{i j}^{(1)}=\left.\frac{\partial^{i+j} g^{(1)}}{\partial^{i} x \partial^{j} y}\right|_{\left(x_{0}, y_{0}, \lambda_{1}\right)}, \quad g_{i j}^{(2)}=\left.\frac{\partial^{i+j} g^{(2)}}{\partial^{i} x \partial^{j} y}\right|_{\left(x_{0}, y_{0}, \lambda_{2}\right)}, \\
g^{(1)}=r x\left(1-\frac{x}{K_{0}}\right)-\frac{x y}{a_{0}+x^{2}}-r \lambda_{1} x^{2}, \quad g^{(2)}=y\left(\frac{\mu_{0} x}{a_{0}+x^{2}}-D_{0}-\lambda_{2}\right) .
\end{gathered}
$$


Decompose the enlarged phase space $B C$ of system (4.20) as $B C=P \oplus \operatorname{Ker} \pi$. Then $y$ in system (4.20) can be decomposed as $y=\Phi z+u$ with $z \in R^{2}$ and $u \in Q^{\prime}$. Hence, system (4.20) is decomposed as follows

$$
\begin{aligned}
& \dot{z}=B_{1}+B_{2} z+\Psi(0) G(\Phi z+u), \\
& \dot{u}=A_{Q^{\prime}} u+(I-\pi) X_{0}\left[B_{0}+B_{2}(\Phi(0) z+u(0))+G(\Phi z+u)\right],
\end{aligned}
$$

where

$$
\begin{aligned}
X_{0}(\theta) & = \begin{cases}I, & \theta=0 \\
0, & -1 \leq \theta<0\end{cases} \\
B_{0}=\left(\begin{array}{c}
-r x_{0}^{2} \tau \lambda_{1} \\
-\tau y_{0} \lambda_{2}
\end{array}\right), \quad B_{1} & =\Psi(0) B_{0}, \quad B_{2}=\left(\begin{array}{cc}
-2 r \tau x_{0} \lambda_{1} & 1 \\
0 & -\tau \lambda_{2}
\end{array}\right),
\end{aligned}
$$

and

$$
G(\phi)=\left(\begin{array}{c}
\sum_{i+j \geq 2} \frac{1}{i ! j !} \tau g_{i j}^{(1)}\left[\phi_{1}(0)\right]^{i}\left[\phi_{2}(0)\right]^{j} \\
\sum_{i+j \geq 2} \frac{1}{i ! j !} \tau g_{i j}^{(2)}\left[\phi_{1}(-1)\right]^{i}\left[\phi_{2}(0)\right]^{j}
\end{array}\right),
$$

here $\phi=\left(\phi_{1}, \phi_{2}\right)$.

To compute the normal form of system (4.20) at $\left(x_{0}, y_{0}\right)$, consider

$$
\dot{z}=B_{1}+B_{2} z+\Psi(0) G(\Phi z)
$$

that is,

$$
\begin{aligned}
& \dot{z}_{1}=-r \tau a_{0} \lambda_{1}-2 r \tau x_{0} \lambda_{1} z_{1}+z_{2}-\left(r \tau \lambda_{1}+\frac{r \tau D_{0}}{2 \mu_{0}}\right) z_{1}^{2}+\tau R_{1}\left(z_{1}, z_{2}\right), \\
& \dot{z}_{2}=\frac{1}{2} r \tau^{2} x_{0} \lambda_{2}-\tau \lambda_{2} z_{2}+\frac{1}{2} \frac{r \tau^{2} D_{0}^{2}}{\mu_{0}}\left(z_{1}-z_{2}\right)^{2}+\tau R_{2}\left(z_{1}, z_{2}\right),
\end{aligned}
$$

where $R_{1}$ and $R_{2}$ are $C^{\infty}$ functions in $\left(z_{1}, z_{2}\right)$ at least of the third order. Following the procedure of deriving normal form in Kuznetsov [57], system (4.22) can be reduced to

$$
\begin{aligned}
& \dot{z}_{1}=z_{2}, \\
& \dot{z}_{2}=\gamma_{1}+\gamma_{2} z_{2}+\alpha z_{1}^{2}+\beta z_{1} z_{2}+R\left(z_{1}, z_{2}, \gamma_{1}, \gamma_{2}\right),
\end{aligned}
$$

here $\gamma_{1}=\frac{1}{2} r \tau^{2} x_{0} \lambda_{2}, \gamma_{2}=-\tau \lambda_{2}+\frac{r \tau x_{0}}{2}\left(r \tau^{2} D_{0}-4\right) \lambda_{1}$, and $R=O\left(|\gamma|^{2}\right)+O\left(|\gamma z|^{3}\right)$.

Hence, when $\tau^{2} r D_{0} \neq 4$, system (4.19) exhibits Bogdanov-Takens bifurcation (Xiao and Ruan [92]).

Theorem 30. When $\tau^{2} r D_{0} \neq 4$, there exists a unique smooth curve $H L$ corresponding to homoclinic bifurcation and a unique smooth curve $H$ corresponding to Hopf bifurcation, such that system (4.19) has a unique and hyperbolic stable cycle for parameter values inside the region bounded by $H$ and $H L$ in the lower half plane $\lambda_{2}<0$ and no cycles outside this region. The local representations of these bifurcations curves are given by

$$
H L=\left\{\left(\lambda_{1}, \lambda_{2}\right) ; \quad \gamma_{2}-\frac{5}{7} \beta \sqrt{-\frac{\gamma_{1}}{\alpha}}=0, \quad \gamma_{1}<0\right\}
$$


and

$$
H=\left\{\left(\lambda_{1}, \lambda_{2}\right) ; \quad \gamma_{2}-\beta \sqrt{-\frac{\gamma_{1}}{\alpha}}=0, \quad \gamma_{1}<0\right\}
$$

\section{Predator-Prey Models with Delay and Harvesting}

In this subsection we review some results in Martin and Ruan [63] and Xia et al. [89] about the combined effects of constant-rate harvesting and delay on the dynamics of predator-prey systems. Firstly, we consider a generalized Gause-type predator-prey model with prey harvesting and a time delay in the predator response function. Secondly, a generalized Gause-type predator-prey model with prey harvesting and a time delay in the prey specific growth term is analyzed. Thirdly, we study the Wangersky-Cunningham predator-prey model with prey harvesting. Finally, we analyze a generalized Gause-type predator-prey model with predator harvesting and a time delay in the predator response function.

\subsection{Gause Models with Prey Harvesting and Delay in the Predator Re- sponse}

In this section, we consider the system

$$
\begin{aligned}
& x^{\prime}(t)=x(t) g(x(t))-y(t) p(x(t))-H, \\
& y^{\prime}(t)=y(t)[-d+\mu p(x(t-\tau))],
\end{aligned}
$$

where $\mu>0$ is the rate of conversion of consumed prey to predator, $d>0$ is the death rate of the predator in the absence of the prey, $H$ is the constant-rate harvesting of the prey species $x$. Also, $g(x)$ is the specific growth rate of the prey in the absence of predators where $g(0) \geq 0$ and $g(x)$ is continuous and decreasing in $x$. The capture rate of prey per predator, that is the functional response is given by $p(x)$ where $p(x)>0$ and $p^{\prime}(x)>0$. The delay $\tau \geq 0$ is a constant.

When $H=0$, the system has been studied by many researchers, see Beretta and Kuang [7], Ruan [74] and the references cited therein.

The positive equilibrium $\left(x^{*}, y^{*}\right)$ is given by

$$
x^{*}=p^{-1}\left(\frac{d}{\mu}\right), y^{*}=\frac{d}{\mu}\left[x^{*} g\left(x^{*}\right)-H\right] .
$$

The $y^{*}$ value implies that $x^{*} g\left(x^{*}\right)>H$. Let $X=x-x^{*}, Y=y-y^{*}$. We then obtain the linearized system

$$
\begin{aligned}
& X^{\prime}(t)=\left[g\left(x^{*}\right)+x^{*} g^{\prime}\left(x^{*}\right)-y^{*} p^{\prime}\left(x^{*}\right)\right] X(t)-p\left(x^{*}\right) Y(t) \\
& Y^{\prime}(t)=\mu y^{*} p^{\prime}(x) X(t-\tau) .
\end{aligned}
$$

The characteristic equation takes the form

$$
\lambda^{2}+p \lambda+r e^{-\lambda \tau}=0,
$$


where

$$
p=-\left[g\left(x^{*}\right)+x^{*} g^{\prime}\left(x^{*}\right)-y^{*} p^{\prime}\left(x^{*}\right)\right], \quad r=\mu x^{*} y^{*} p\left(x^{*}\right) p^{\prime}\left(x^{*}\right) .
$$

Define

$$
\omega_{ \pm}^{2}=\frac{-p^{2} \pm \sqrt{p^{4}+4 r^{2}}}{2}
$$

and

$$
\tau_{j}^{+}=\frac{1}{\omega_{+}} \arctan \left(\frac{p}{\omega_{+}}\right)+\frac{2 j \pi}{\omega_{+}}, \quad j=0,1,2, \ldots
$$

By Theorem 7, we have the following result (Martin and Ruan [63]).

Corollary 31. Let $\tau_{j}^{+}$be defined by equation (5.5). If $p>0$ and $r>0$, then the equilibrium $\left(x^{*}, y^{*}\right)$ of system (5.1) is stable for $\tau<\tau_{0}^{+}$and unstable for $\tau>\tau_{0}^{+}$. The system undergoes Hopf bifurcation at $\tau_{0}^{+}$.

Example 32. As an example, we consider the system

$$
\begin{aligned}
& \frac{d x}{d t}=x(t)\left\{2\left[1-\frac{x(t)}{50}\right]-\frac{y(t)}{x(t)+40}\right\}-10 \\
& \frac{d y}{d t}=y(t)\left[-3+\frac{6 x(t-\tau)}{x(t-\tau)+40}\right]
\end{aligned}
$$

The positive equilibrium is $\left(x^{*}, y^{*}\right)=(40,12)$. By Corollary 31 , there is a critical value $\tau_{0}=$ 8.205. The equilibrium $\left(x^{*}, y^{*}\right)=(40,12)$ is asymptotically stable for $\tau<8.205$, becomes unstable for $\tau>8.205$, and there is a bifurcating periodic solution. Figure 1 shows that the equilibrium

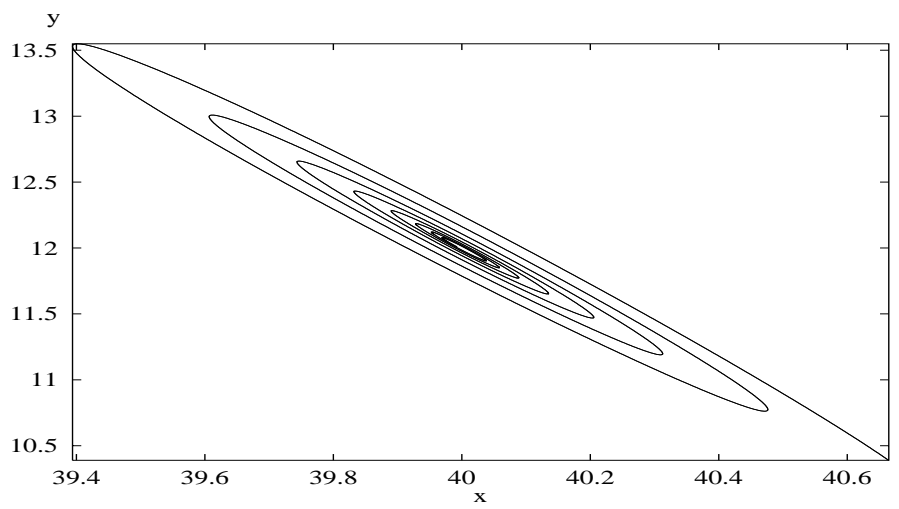

Figure 1: The equilibrium $\left(x^{*}, y^{*}\right)=(40,12)$ of system (5.6) is an asymptotically stable focus for $\tau=7$.

point $(40,12)$ is a stable focus for $\tau=7$ and Figure 2 shows that for $\tau=9$ a limit cycle is present. 


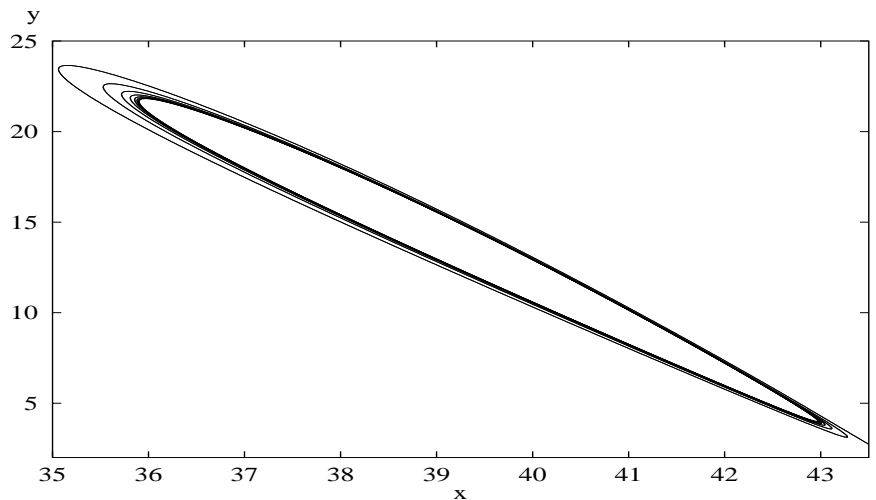

Figure 2: There is a bifurcating periodic solution of system (5.6) for $\tau=9$.

We can also vary the harvesting constant $H$ to see how it affects the dynamics. Notice that the equilibrium value $y^{*}$ depends on $H$ : the more a prey population is harvested, the lower is the number of predators at the equilibrium, and the less a prey population is harvested, the higher is the number of predators $y^{*}$. In this example, the critical harvesting rate is $H=x g(x)=16$. Therefore, for $H<16$ the equilibrium is positive and stable, but for $H \geq 16$ the prey population is driven to extinction and the system collapses. Thus, a variation in $H$ can change the stability of the model (5.6). For example, choose $\tau=9$ (a value at which the equilibrium is unstable),
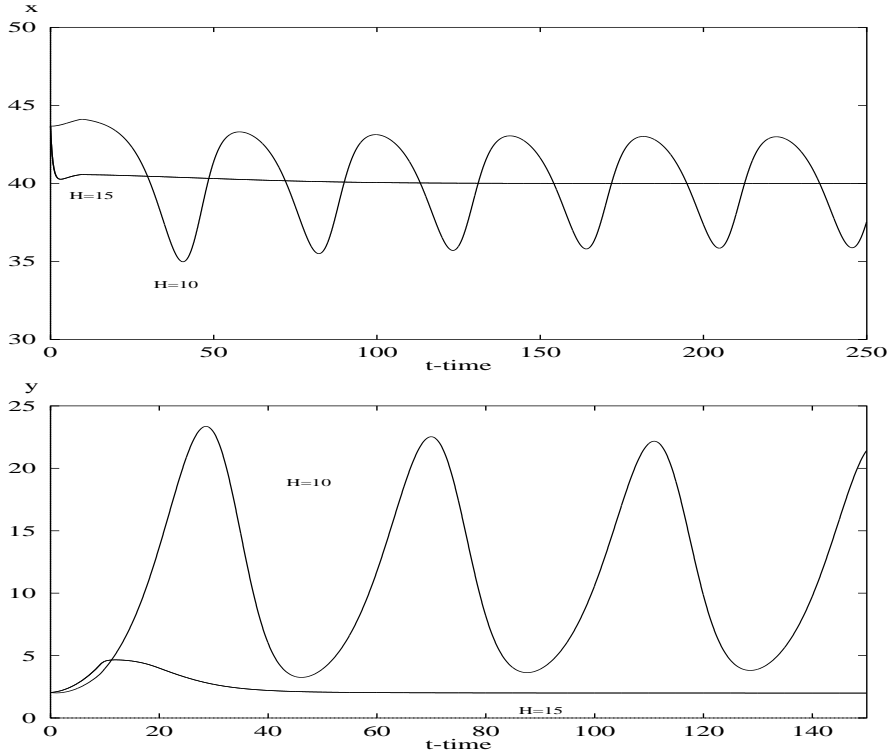

Figure 3: Behaviors of the prey and predator populations of system (5.6) for different values of $H$ with $\tau=9$.

when $H=10$ both the prey and predator populations oscillate about the equilibrium values; when $H=15$ (a value close to the critical harvesting rate) both the prey and predator populations converge to the equilibrium values (see Figure 3). Therefore, the system regains its stability when 
the prey harvesting constant is increased but less than the critical harvesting. This indicates that the harvesting rate has an effect of stabilizing the equilibrium of the model.

\subsection{Gause Models with Prey Harvesting and Delayed Prey Specific Growth}

Consider the system

$$
\begin{aligned}
& x^{\prime}(t)=x(t) g(x(t-\tau))-y(t) p(x(t))-H, \\
& y^{\prime}(t)=y(t)[-d+\mu p(x(t))] .
\end{aligned}
$$

When $\tau=0$, the ODE model was studied by Brauer and Soudack $[14,15]$; when $H=0$, the delayed predator-prey model has been analyzed by May [61], Hassard et al. [48], Ruan [74], etc. The stability of system (5.7) was investigated by Brauer [17].

The positive equilibrium $E^{*}=\left(x^{*}, y^{*}\right)$ has the same expression as that for model (5.1). The linearized system about the equilibrium point is given by

$$
\begin{aligned}
& X^{\prime}(t)=x^{*} g^{\prime}\left(x^{*}\right) X(t-\tau)+\left(g\left(x^{*}\right)-y^{*} p^{\prime}\left(x^{*}\right)\right) X(t)-p\left(x^{*}\right) Y(t), \\
& Y^{\prime}(t)=\mu y^{*} p^{\prime}\left(x^{*}\right) X(t) .
\end{aligned}
$$

The characteristic equation is

$$
\lambda^{2}+p \lambda+q \lambda e^{-\lambda \tau}+\alpha=0
$$

where

$$
p=-\left[g\left(x^{*}\right)-y^{*} p^{\prime}\left(x^{*}\right)\right], q=-x^{*} g^{\prime}\left(x^{*}\right), \alpha=\mu y^{*} p\left(x^{*}\right) p^{\prime}\left(x^{*}\right) .
$$

Define

$$
\omega_{ \pm}^{2}=\frac{q^{2}-p^{2}+2 \alpha \pm \sqrt{\left(q^{2}-p^{2}+2 \alpha\right)^{2}-4 \alpha^{2}}}{2}
$$

and

$$
\tau_{j}^{ \pm}=\frac{1}{\omega_{ \pm}} \arctan \left(\frac{\omega_{ \pm}^{2}-\alpha}{p \omega_{ \pm}}\right)+\frac{2 j \pi}{\omega_{ \pm}}, \quad j=0,1,2, \ldots
$$

Applying Theorem 7, we have the following theorem (Martin and Ruan [63]).

Corollary 33. Let $\tau_{j}^{ \pm}$be defined by equation (5.11).

(i) If $p+q>0, \alpha>0$ and $p^{2}-q^{2}-2 \alpha>0$, then the equilibrium ( $\left.x^{*}, y^{*}\right)$ of system (5.7) is asymptotically stable for all $\tau \geq 0$.

(ii) If $p+q>0, \alpha>0, q^{2}-p^{2}+2 \alpha>0$ and $\left(q^{2}-p^{2}+2 \alpha\right)^{2}=4 \alpha^{2}$, then the equilibrium $\left(x^{*}, y^{*}\right)$ of system (5.7) is asymptotically stable for $\tau \in\left[0, \tau_{0}\right)$ and unstable for $\tau>\tau_{0}$. Hopf bifurcation occurs when $\tau=\tau_{0}$; that is, a family of periodic solutions bifurcates from $\left(x^{*}, y^{*}\right)$ as $\tau$ passes through the critical value $\tau^{*}$. 
(iii) If $p+q>0, \alpha>0, q^{2}-p^{2}+2 \alpha>0$ and $\left(q^{2}-p^{2}+2 \alpha\right)^{2}>4 \alpha^{2}$, then there exists a positive integer $k$ such that there are $k$ switches from stability to instability and to stability. In other words, when

$$
\tau \in\left[0, \tau_{0}^{+}\right),\left(\tau_{0}^{-}, \tau_{1}^{+}\right), \ldots,\left(\tau_{k-1}^{-}, \tau_{k}^{+}\right),
$$

the equilibrium $\left(x^{*}, y^{*}\right)$ of system (5.7) is stable, and when

$$
\tau \in\left[\tau_{0}^{+}, \tau_{0}^{-}\right),\left(\tau_{1}^{+}, \tau_{1}^{-}\right), \ldots,\left(\tau_{k-1}^{+}, \tau_{k}^{-}\right)
$$

$\left(x^{*}, y^{*}\right)$ is unstable. Therefore, there are bifurcations at $\left(x^{*}, y^{*}\right)$ for $\tau=\tau_{j}^{ \pm}, j=0,1,2, \ldots$

Example 34. As an example, consider the system

$$
\begin{aligned}
& \frac{d x}{d t}=x(t)\left\{2\left[1-\frac{x(t-\tau)}{40}\right]-\frac{y(t)}{x(t)+10}\right\}-10, \\
& \frac{d y}{d t}=y(t)\left[\frac{x(t)}{x(t)+10}-\frac{2}{3}\right] .
\end{aligned}
$$

There is a positive equilibrium $\left(x^{*}, y^{*}\right)=(20,15)$. By Corollary 33, there is a critical value $\tau_{0}=$ 0.8256 , the equilibrium $\left(x^{*}, y^{*}\right)$ is stable when $\tau<0.8256$. A Hopf bifurcation occurs when $\tau=0.8256$; and the equilibrium becomes unstable and a bifurcating periodic solution exists when $\tau>0.8256$. Figure 4 shows that both the prey and predator populations reach periodic oscillations around the equilibrium $\left(x^{*}, y^{*}\right)=(20,15)$ in finite time.
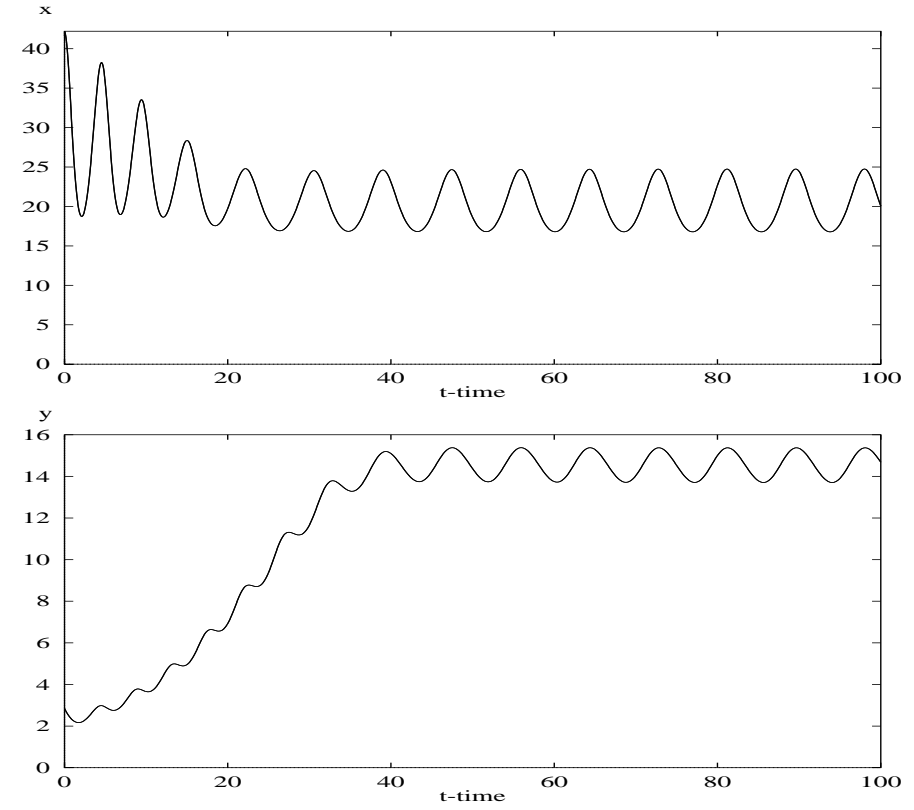

Figure 4: The oscillations of the prey and predator populations in time for $\tau=0.826$ 
We can also see that varying $H$ will affect the dynamics of the model. For $\tau=0.826$, varying the value of the harvesting constant $H$ changes the $y^{*}$ value of the equilibrium point $\left(x^{*}, y^{*}\right)$ : increasing $H$ decreases $y^{*}$ but does not change the frequencies of these oscillations (see Figure 5). However, as in Example 32, increasing $H$ further can decrease $y^{*}$ to reach zero and thus drive the species to extinction.

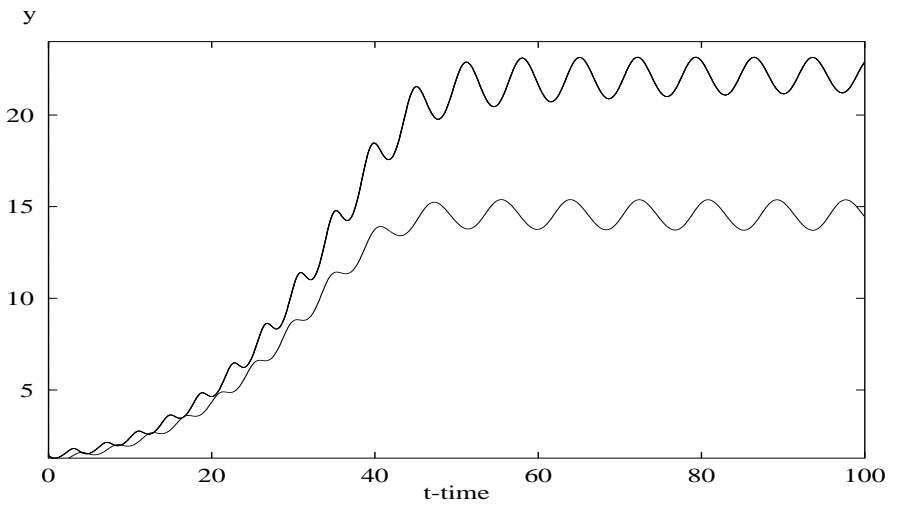

Figure 5: Behavior of the predator population for different values of $H=10$ (top ) and $H=15$ (bottom) for $\tau=0.826$.

\subsection{Wangersky-Cunningham Model with Prey Harvesting}

Consider the system:

$$
\begin{aligned}
& x^{\prime}(t)=x(t)\left[r_{1}-a x(t)-b y(t)\right]-H, \\
& y^{\prime}(t)=-r_{2} y(t)+c x(t-\tau) y(t-\tau),
\end{aligned}
$$

where $r_{1}$ is the rate of increase of the prey population, $r_{2}$ is the death rate of the predator population, $b$ is the coefficient of effect of predation on $x, c$ is the coefficient of effect of predation on $y, H$ is the constant-rate harvesting of the prey species $x$. Also, $a=r_{1} / K_{x}$ where $K_{x}$, a density-dependent term, represents the limitation upon the growth of the prey other than by predation. The delay $\tau \geq 0$ is a constant based on the assumption that the change rate of predators depends on the number of prey and of predators present at some previous time.

The model when $H=0$ was proposed and analyzed by Wangersky and Cunningham [86] and their analysis was criticized by Goel et al. [41]. However, as pointed out by Nunney [71], the analysis of Goel et al. is incomplete either. Notice that our results hold if $H=0$.

The interior equilibrium $\left(x^{*}, y^{*}\right)$ is given by

$$
x^{*}=\frac{r_{2}}{c}, \quad y^{*}=\frac{c r_{1} r_{2}-a r_{2}^{2}-H c^{2}}{b c r_{2}}
$$

if $c r_{1} r_{2}-a r_{2}^{2}-H c^{2} \geq 0$. We can see that as $H$ increases, $y^{*}$ decreases continuously until it reaches zero at the critical harvest rate

$$
H=x^{*}\left(x^{*} r_{1}-a x^{*}\right) .
$$


Let $X=x-x^{*}, Y=y-y^{*}$. We obtain the linearized system

$$
\begin{aligned}
& X^{\prime}(t)=\left(r_{1}-2 a x^{*}-b y^{*}\right) X(t)-b x^{*} Y(t), \\
& Y^{\prime}(t)=c y^{*} X(t-\tau)-r_{2} Y(t)+c x^{*} Y(t-\tau) .
\end{aligned}
$$

The characteristic equation is

$$
\lambda^{2}+p \lambda+r+(s \lambda+q) e^{-\lambda \tau}=0
$$

where

$$
\begin{array}{ll}
p=r_{2}-r_{1}+2 a x^{*}+b y^{*}, & q=c x^{*}\left(r_{1}-2 a x^{*}-b y^{*}\right)+b c x^{*} y^{*}, \\
r=-r_{2}\left(r_{1}-2 a x^{*}-b y^{*}\right), & s=-c x^{*} .
\end{array}
$$

Define

$$
\omega_{ \pm}^{2}=\frac{s^{2}-p^{2}+2 r \pm \sqrt{\left(s^{2}-p^{2}+2 r\right)^{2}-4\left(r^{2}-q^{2}\right)}}{2}
$$

and

$$
\tau_{j}^{ \pm}=\frac{1}{\omega_{ \pm}} \arctan \left(\frac{\omega_{ \pm}\left(p q-r s+s \omega_{ \pm}^{2}\right)}{p s \omega_{ \pm}^{2}+\left(r-\omega_{ \pm}^{2}\right) q}\right)+\frac{2 j \pi}{\omega_{ \pm}}, j=0,1,2, \ldots
$$

By Theorem 7, we have the following theorem (Martin and Ruan [63]).

Corollary 35. Let $\tau_{j}^{ \pm}$be defined by equation (5.18).

(i) If $p+s>0, q+r>0, p^{2}-s^{2}-2 r>0$, and $r^{2}-q^{2}>0$, then the equilibrium $\left(x^{*}, y^{*}\right)$ of system (5.13) is asymptotically stable for all $\tau \geq 0$.

(ii) If $p+s>0, q+r>0$, and $r^{2}-q^{2}<0$, then the equilibrium $\left(x^{*}, y^{*}\right)$ of system (5.13) is asymptotically stable for $\tau<\tau_{0}$ and unstable for $\tau>\tau_{0}$. Hopf bifurcation occurs when $\tau=\tau_{0}$.

(iii) If $p+s>0, q+r>0, r^{2}-q^{2}>0, s^{2}-p^{2}+2 r>0$, and $\left(s^{2}-p^{2}+2 r\right)^{2}>4\left(r^{2}-q^{2}\right)$, then there exists a positive integer $k$ such that there are $k$ switches from stability to instability and to stability. In other words, when

$$
\tau \in\left[0, \tau_{0}^{+}\right),\left(\tau_{0}^{-}, \tau_{1}^{+}\right), \ldots,\left(\tau_{k-1}^{-}, \tau_{k}^{+}\right),
$$

the equilibrium $\left(x^{*}, y^{*}\right)$ of system (5.13) is stable, and when

$$
\tau \in\left[\tau_{0}^{+}, \tau_{0}^{-}\right),\left(\tau_{1}^{+}, \tau_{1}^{-}\right), \ldots,\left(\tau_{k-1}^{+}, \tau_{k}^{-}\right),
$$

$\left(x^{*}, y^{*}\right)$ is unstable. Therefore, there are bifurcations at $\left(x^{*}, y^{*}\right)$ when $\tau=\tau_{j}^{ \pm}, j=0,1,2, \ldots$ 
Example 36. As an example, we consider the following system

$$
\begin{aligned}
& \frac{d x}{d t}=x(t)[20-x(t)-y(t)]-7, \\
& \frac{d y}{d t}=-15 y(t)+3 x(t-\tau) y(t-\tau),
\end{aligned}
$$

which has a positive equilibrium $\left(x^{*}, y^{*}\right)=(5,68 / 5)$. By Corollary 35 there is a critical value $\tau_{0}=0.0385$, when $\tau<0.0385$ the equilibrium $(5,68 / 5)$ is asymptotically stable; when $\tau=0.0385$ the equilibrium $(5,68 / 5)$ loses its stability; and when $\tau>0.0385$ the equilibrium $(5,68 / 5)$ becomes unstable and there is a bifurcating periodic solution (see Figure 6).

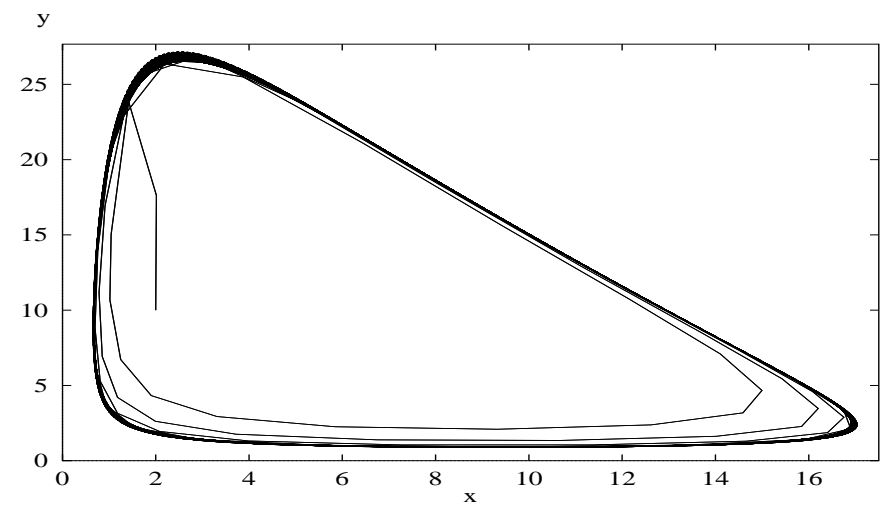

Figure 6: There is a bifurcating periodic solution for $\tau=0.05$.

To see whether varying $H$ will affect the dynamics of the model, we calculate that for $H=1$, $y^{*}=74 / 5$, compared to $y^{*}=68 / 5$ for $H=7$. Therefore, increasing $H$ will decrease $y^{*}$ but does not change the frequency of the oscillations (Figure 7). However, once again increasing $H$ further will decrease $y^{*}$ to zero.

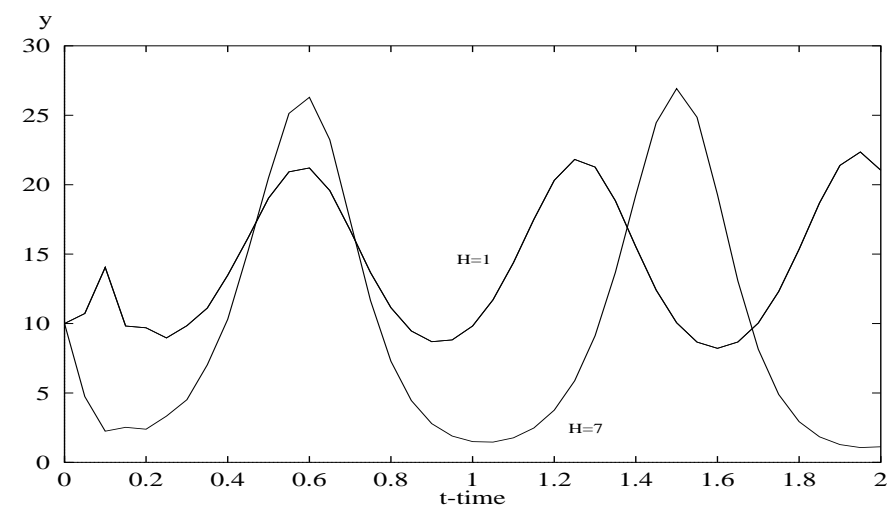

Figure 7: Behavior of the predator population for different values of $H$ for $\tau=0.05$. 


\subsection{Predator Harvesting and Delayed Predator Response}

Finally, we consider a predator-prey model with Holling II functional response in which the predator population is harvested at a constant rate and there is a delay in the predator response term (see Xia et al. [89]):

$$
\left\{\begin{array}{l}
\frac{d x}{d t}=r x(t)\left(1-\frac{x(t)}{K}\right)-\frac{x(t) y(t)}{A+x(t)} \\
\frac{d y}{d t}=y(t)\left(-D+\frac{x(t-\tau)}{A+x(t-\tau)}\right)-H .
\end{array}\right.
$$

When $\tau=0$, system (5.20) was studied in details by Xiao and Ruan [91]. We know that system (5.20) has a unique interior equilibrium $E=\left(x_{0}, y_{0}\right)$ provided that

$$
\left(D-1+\frac{A D}{K}\right)^{2}-4 \frac{(1-D)(A D r+H)}{K r}=0 \quad \text { and } \quad K>\frac{A D}{1-D}
$$

hold and $\left(x_{0}, y_{0}\right)$ is given by

$$
x_{0}=\frac{K(1-D)-A D}{2(1-D)}, \quad y_{0}=r\left(1-\frac{x_{0}}{K}\right)\left(A+x_{0}\right) .
$$

We assume throughout this section that $0<D<1$.

We translate the equilibrium $\left(x_{0}, y_{0}\right)$ of system (5.20) to the origin. Setting $z_{1}(t)=x(t)-$ $x_{0}, z_{2}(t)=y(t)-y_{0}$, system (5.20) can be written as the following system

$$
\left\{\begin{array}{l}
\dot{z}_{1}(t)=\alpha_{1} z_{1}(t)+\alpha_{2} z_{2}(t)+\sum_{i+j \geq 2} \frac{1}{i ! j !} f_{i j}^{(1)} z_{1}^{i}(t) z_{2}^{j}(t) \\
\dot{z}_{2}(t)=\beta_{1} z_{1}(t-\tau)+\beta_{2} z_{2}(t)+\sum_{i+j \geq 2} \frac{1}{i ! j !} f_{i j}^{(2)} z_{1}^{i}(t-\tau) z_{2}^{j}(t),
\end{array}\right.
$$

where

$$
\begin{aligned}
\alpha_{1} & =r-\frac{2 x_{0} r}{K}-\frac{A y_{0}}{\left(A+x_{0}\right)^{2}}, \quad \alpha_{2}=-\frac{x_{0}}{A+x_{0}}, \\
\beta_{1} & =\frac{A y_{0}}{\left(A+x_{0}\right)^{2}}, \quad \beta_{2}=-D+\frac{x_{0}}{A+x_{0}}, \\
f_{i j}^{(1)} & =\left.\frac{\partial^{i+j} f^{(1)}}{\partial x^{i} \partial y^{j}}\right|_{\left(x_{0}, y_{0}\right)}, \quad f_{i j}^{(2)}=\left.\frac{\partial^{i+j} f^{(2)}}{\partial x^{i} \partial y^{j}}\right|_{\left(x_{0}, y_{0}\right)}, \quad i, j \geq 0, \\
f^{(1)} & =r x\left(1-\frac{x}{K}\right)-\frac{x y}{A+x}, \quad f^{(2)}=y\left(-D+\frac{x}{A+x}\right)-H .
\end{aligned}
$$

Consider the linearized system of (5.22) at the zero equilibrium

$$
\left\{\begin{array}{l}
\dot{z}_{1}(t)=\alpha_{1} z_{1}(t)+\alpha_{2} z_{2}(t) \\
\dot{z}_{2}(t)=\beta_{1} z_{1}(t-\tau)+\beta_{2} z_{2}(t) .
\end{array}\right.
$$

The characteristic equation for system (5.23) takes the form

$$
\lambda^{2}-\lambda\left(\alpha_{1}+\beta_{2}\right)+\alpha_{1} \beta_{2}-\alpha_{2} \beta_{1} e^{-\lambda \tau}=0 .
$$


By means of the software Maple, we can compute $\alpha_{1} \beta_{2}-\alpha_{2} \beta_{1}=0$. We can see that (5.24) has two zero eigenvalues and no other eigenvalues if and only if $\tau=\frac{\alpha_{1}+\beta_{2}}{\alpha_{2} \beta_{1}}$ and $\tau \neq \sqrt{\frac{2}{\alpha_{2} \beta_{1}}}$. Because $\tau>0$ and $\alpha_{2} \beta_{1}<0$, we know that $\tau \neq \sqrt{\frac{2}{\alpha_{2} \beta_{1}}}$.

Normalizing the delay $\tau$ in system (5.20) by scaling the time $t \rightarrow t / \tau$, system (5.20) is transformed into

$$
\left\{\begin{array}{l}
\dot{x}(t)=\tau\left[r x(t)\left(1-\frac{x(t)}{K}\right)-\frac{x(t) y(t)}{A+x(t)}\right] \\
\dot{y}(t)=\tau y(t)\left[-D+\frac{x(t-1)}{A+x(t-1)}\right]-\tau H .
\end{array}\right.
$$

Setting $z_{1}(t)=x(t)-x_{0}, z_{2}(t)=y(t)-y_{0}$, system (5.25) can be rewritten as a functional differential equation in $C:=C\left([-1,0], \mathbb{R}^{2}\right)$

$$
\left\{\begin{array}{l}
\dot{z}_{1}(t)=\tau\left[\alpha_{1} z_{1}(t)+\alpha_{2} z_{2}(t)+\sum_{i+j \geq 2} \frac{1}{i ! j !} f_{i j}^{(1)} z_{1}^{i}(t) z_{2}^{j}(t)\right], \\
\dot{z}_{2}(t)=\tau\left[\beta_{1} z_{1}(t-1)+\beta_{2} z_{2}(t)+\sum_{i+j \geq 2} \frac{1}{i ! j !} f_{i j}^{(2)} z_{1}^{i}(t-1) z_{2}^{j}(t)\right] .
\end{array}\right.
$$

Using the normal form theory developed by Faria and Magalhães [32, 34], following the techniques in Xiao and Ruan [92] or in section 4, we obtain that the normal form for (5.26) is as follows (see Xia et al. [89])

$$
\left\{\begin{array}{l}
\dot{x}_{1}=x_{2}+O\left(\left|\left(x_{1}, x_{2}\right)\right|^{3}\right) \\
\dot{x}_{2}=B_{1} x_{1}^{2}+B_{2} x_{1} x_{2}+O\left(\left|\left(x_{1}, x_{2}\right)\right|^{3}\right)
\end{array}\right.
$$

where

$$
B_{1}=\frac{1}{2} \tau \alpha_{2}^{2}\left(e f_{20}^{(1)}+f f_{20}^{(2)}\right)-\tau \alpha_{1} \alpha_{2}\left(e f_{11}^{(1)}+f f_{11}^{(2)}\right)
$$

$B_{2}=\tau \alpha_{2}^{2}\left(g f_{20}^{(1)}+h f_{20}^{(2)}+e f_{20}^{(1)}\right)-\tau \alpha_{1} \alpha_{2}\left(2 g f_{11}^{(1)}+2 e f_{11}^{(1)}+2 h f_{11}^{(2)}+f f_{11}^{(2)}\right)+\tau \tau_{0}^{-1} \alpha_{2}\left(e f_{11}^{(1)}+f f_{11}^{(2)}\right)$ and $e, f, g, h$ satisfy the following equations

$$
\left\{\begin{array}{l}
\alpha_{2} e+\beta_{2} f=0 \\
\tau_{0} \alpha_{2} g+\tau_{0} \beta_{2} h=f \\
h\left(\alpha_{1}-\tau_{0} \alpha_{2} \beta_{1}\right)-\alpha_{2} g+\frac{1}{2} \tau_{0} \alpha_{2} \beta_{1} f=1 \\
h\left(\alpha_{1}-\tau_{0}^{-1}-\frac{1}{2} \tau_{0} \beta_{1} \alpha_{2}\right)-\alpha_{2} g+\frac{1}{3} \tau_{0} \beta_{1} \alpha_{2} f=0 \\
-e \alpha_{2}+f\left(\alpha_{1}-\tau_{0}^{-1}-\frac{1}{2} \tau_{0} \beta_{1} \alpha_{2}\right)=1
\end{array}\right.
$$

The above arguments imply the following result (Xia et al. [89]).

Theorem 37. Suppose that (5.21) holds. Then the equilibrium $\left(x_{0}, y_{0}\right)$ of system (5.20) is a Bogdanov-Takens singularity when $\tau=\frac{\alpha_{1}+\beta_{2}}{\alpha_{2} \beta_{1}}$. 
Next, we are interested in determining a versal unfolding for system (5.20) with the BogdanovTakens singularity $\left(x_{0}, y_{0}\right)$. Note that $\alpha_{1} \beta_{2}-\alpha_{2} \beta_{1}=0$. We introduce two bifurcation parameters $\mu=\left(\mu_{1}, \mu_{2}\right)$ by setting $\tau=\tau_{0}+\mu_{1}, \beta_{1}=\frac{\alpha_{1} \beta_{2}}{\alpha_{2}}+\mu_{2}$. System (5.26) is rewritten as

$$
\dot{z}(t)=L_{0}\left(z_{t}\right)+L_{1}(\mu) z_{t}+\tilde{F}\left(z_{t}, \mu\right)
$$

where

$$
\begin{gathered}
L_{1}(\mu) \varphi=\mu_{1} L(\varphi)+\tau_{0} \mu_{2}\left(\begin{array}{c}
0 \\
\varphi_{1}(-1)
\end{array}\right) \\
\tilde{F}(\varphi, \mu)=\mu_{1} \mu_{2}\left(\begin{array}{c}
0 \\
\varphi_{1}(-1)
\end{array}\right)+\left(\tau_{0}+\mu_{1}\right) F(\varphi) \text { for } \varphi=\left(\begin{array}{l}
\varphi_{1} \\
\varphi_{2}
\end{array}\right) .
\end{gathered}
$$

Therefore, following the techniques in Xiao and Ruan [92] or in section 4, we obtain the normal form of (5.28) on the center manifold

$$
\left\{\begin{array}{l}
\dot{x}_{1}=x_{2}+\text { h.o.t. } \\
\dot{x}_{2}=\lambda_{1} x_{1}+\lambda_{2} x_{2}+B_{1} x_{1}^{2}+B_{2} x_{1} x_{2}+\text { h.o.t. }
\end{array}\right.
$$

where

$$
\lambda_{1}=-\tau_{0} \alpha_{2} f \mu_{2}, \quad \lambda_{2}=\beta_{2} \alpha_{1} f \mu_{1}-\tau_{0} \alpha_{2} h \mu_{2}
$$

and

$$
\begin{aligned}
B_{1} & =\frac{\tau_{0}}{2}\left[\alpha_{2}^{2}\left(e f_{20}^{(1)}+f f_{20}^{(2)}\right)-2 \alpha_{1} \alpha_{2}\left(e f_{11}^{(1)}+f f_{11}^{(2)}\right)\right], \\
B_{2} & =\tau_{0}\left\{\alpha_{2}^{2}\left(g f_{20}^{(1)}+h f_{20}^{(2)}\right)-2 \alpha_{1} \alpha_{2}\left(g f_{11}^{(1)}+h f_{11}^{(2)}\right)+\alpha_{2}^{2} e f_{20}^{(1)}\right. \\
& \left.-\alpha_{2}\left[\left(2 \alpha_{1}-\tau_{0}^{-1}\right) f_{11}^{(1)} e+\left(\alpha_{1}-\tau_{0}^{-1}\right) f_{11}^{(2)} f\right]\right\} .
\end{aligned}
$$

We have the following result on Bogdanov-Takes bifurcation (see Xia et al. [89]).

Theorem 38. Let $\mu_{1}, \mu_{2}$ be defined by $\tau=\tau_{0}+\mu_{1}, \beta_{1}=\frac{\alpha_{1} \beta_{2}}{\alpha_{2}}+\mu_{2}$, where $\tau_{0}=\frac{\alpha_{1}+\beta_{2}}{\alpha_{2} \beta_{1}}$. For $\left(x_{0}, y_{0}\right)$ and $\mu_{1}=0, \mu_{2}=0$, system (5.20) exhibits Bogdanov-Takes bifurcation.

As an example, we consider system (5.20) with $r=1, K=2, A=1, D=\frac{1}{3}$, that is,

$$
\left\{\begin{array}{l}
\dot{x}(t)=x(t)\left(1-\frac{x(t)}{2}\right)-\frac{x(t) y(t)}{1+x(t)} \\
\dot{y}(t)=y(t)\left(-\frac{1}{3}+\frac{x(t-\tau)}{1+x(t-\tau)}\right)-H .
\end{array}\right.
$$

Using Theorem 37, we know that system (5.34) has a Bogdanov-Takens singularity point $\left(x_{0}, y_{0}\right)=$ $\left(\frac{5}{4}, \frac{27}{32}\right)$ when $\left(H_{0}, \tau_{0}\right)=\left(\frac{3}{16}, \frac{21}{10}\right)$. Theorem 38 implies that system (5.34) undergoes BogdanovTakens bifurcation when $\tau$ and $H$ vary in a small neighborhood of $\tau_{0}$ and $H_{0}$. Now introduce two bifurcation parameters $\mu_{1}, \mu_{2}$ by setting $\tau=\frac{21}{10}+\mu_{1}, \beta_{1}=\frac{\alpha_{1} \beta_{2}}{\alpha_{2}}+\mu_{2}$, i.e. $H=H_{0}+\mu_{2}$. Following the analysis in this section, we obtain the versal unfolding for system (5.34) as follows

$$
\left\{\begin{array}{l}
\dot{x}_{1}=x_{2}+\text { h.o.t. } \\
\dot{x}_{2}=\lambda_{1} x_{1}+\lambda_{2} x_{2}+B_{1} x_{1}^{2}+B_{2} x_{1} x_{2}+\text { h.o.t. }
\end{array}\right.
$$


where

$$
\begin{aligned}
& \lambda_{1}=-2.034602076 \mu_{2}, \quad \lambda_{2}=0.1614763552 \mu_{1}+1.919613031 \mu_{2}, \\
& B_{1}=0.3767781622, \quad B_{2}=-0.715752274 .
\end{aligned}
$$

Therefore, system (5.34) exhibits Bogdanov-Takens bifurcation when the parameters $\mu_{1}, \mu_{2}$ vary in a small neighborhood of the origin. On the lines $H_{ \pm}$, there exists stable Hopf bifurcation, while there exists curves $H L_{ \pm}$corresponding to homoclinic bifurcation. The bifurcation diagram is depicted in Figure 8.

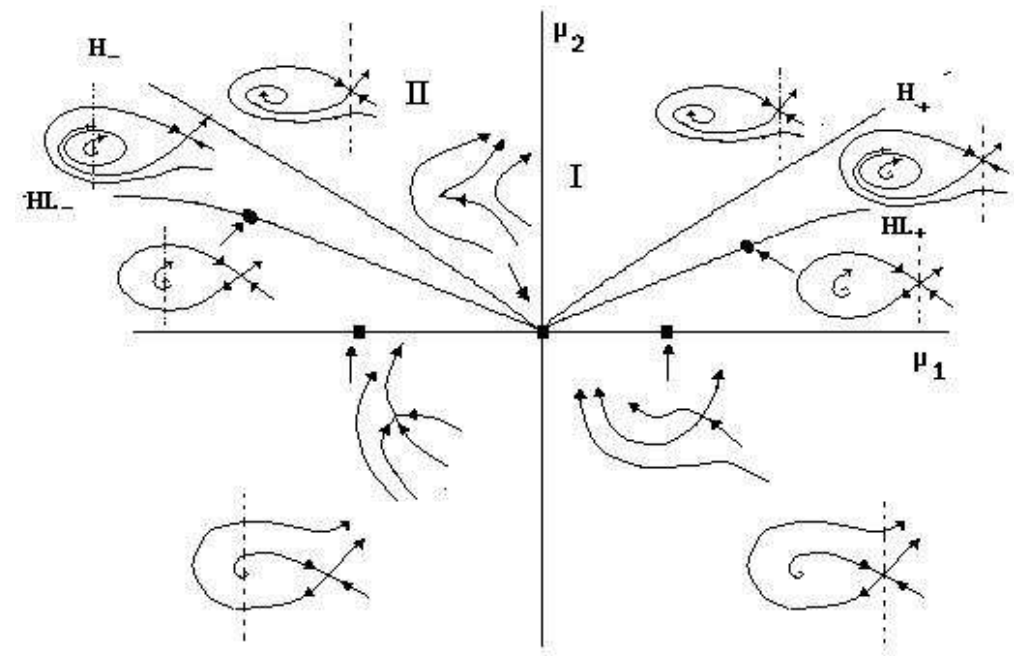

Figure 8: The Bogdanov-Takens bifurcation diagram and phase portraits for system (5.34).

Remark 39. Similarly, we can study Bogdanov-Takens bifurcation in the following predator-prey model with predator harvesting and delayed prey specific growth

$$
\left\{\begin{array}{l}
\frac{d x}{d t}=r x(t)\left(1-\frac{x(t-\tau)}{K}\right)-\frac{x(t) y(t)}{A+x(t)}, \\
\frac{d y}{d t}=y(t)\left(-D+\frac{x(t)}{A+x(t)}\right)-H .
\end{array}\right.
$$

\section{Discussion}

Predator-prey models play a crucial role in studying population dynamics and the management of renewable resources. The effect of constant-rate harvesting or time delay on the dynamics of predator-prey systems has been investigated extensively. Very rich and interesting dynamical behaviors, such as the existence of multiple equilibria, Hopf bifurcation, limit cycles, homoclinic loops, and Bogdanov-Takens bifurcations, have been observed in predator-prey system with time delay or constant-rate harvesting. In this article, I have reviewed some recent results obtained by 
myself and my collaborators on predator-prey system with time delay or with both time delay and constant-rate harvesting.

Basically, time delay can be incorporated into a predator-prey model in four different ways: (i) delayed inter-specific interactions (such as model (3.1)); (ii) delayed predator response (such as model (3.10)); (iii) delayed prey specific growth (such as the May-type model (3.18)); and (iv) delayed predation (such as the Wangersky-Cunningham type model (1.9)). It has been observed that time delay can induce oscillations via Hopf bifurcation in all four types of models. Moreover, May-type and Wangersky-Cunningham-type predator-prey models can exhibit switch of stability when the time delay takes a sequence of critical values. Furthermore, codimension 2 bifurcations can occur in predator-prey models with delayed predator response when the functional response function is nonmonotonic.

Constant-rate harvesting could induce more complex dynamics in delayed predator-prey systems, depending on which species is harvested. When the prey is selectively harvested, the dynamics are similar to that of the models without harvesting and Hopf bifurcation usually occurs. Of course over-harvesting can always drive both species to extinction. On the other hand, constant-rate harvesting on the predators can induce bifurcation on the number of positive equilibria, multiple positive equilibria and degenerate equilibria can exist, and Bogdanov-Takens bifurcation can occur. The codimension 2 bifurcation diagram in the predator-prey models with predator harvesting demonstrates that there are some parameter regions in which both predator and prey species can be driven to extinction, leading to the catastrophe or overexploitation scenario. This indicates that appropriate harvesting of predator population is crucial in the long term survival of both predator and prey species.

Research on the dynamics of predator-prey models with discrete delay is still very active. For example, relative amount of attention has been paid to delayed predator-prey models with stage structure, either in the prey or in predators (Wang and Chen [85], Liu et al. [58], Gourley and Kuang [45], Qu and Wei [72]). Most of these models can be simplified into two-dimensional systems with delay-dependent coefficients which can be treated by the techniques in Beretta and Kuang [9].

From a biological point of view, it will be very interesting and helpful to collaborate with biologists on applying the existing models and results to some biological data. For example, the results on codimension 2 bifurcation in delayed predator-prey models with predator harvesting may provide some explanations for the collapse of the Atlantic cod stocks in the Canadian Grand Banks (Hutchings and Myers [55], Myers et al. [66]) and may be useful in designing fishing policies for the fishery industry (Myers and Worm [67]).

From the point of view of new dynamical behaviors, I think two classes of models worth study. (i) Predator-prey systems with multiple delays (see Freedman and Rao [39], Boese [10] and He [52] for Kolmogorov-type models and Bartlett model (1.8), Hastings [49], Nunney [70, 71], and Ma [61] for non-Kolmogorov-type models). As Nakaoka et al. [69] showed that a LotkaVolterra predator-prey model with delays in the specific growth terms for both species can exhibit chaotic behavior, I expect that other predator-prey models with multiple delays could have similar complex dynamics. (ii) Delayed predator-prey models with both predator and prey harvesting (see Brauer and Soudack [14, 15], Myerscough et al. [68], and Hogarth et al. [53] for some 
ODE models). I suspect that more degenerate bifurcations, such as fold-Hopf, Hopf-Hopf, and degenerate Bogdanov-Takens bifurcations (Kuznetsov [57]), may occur.

\section{Acknowledgments}

The author is very grateful to the two anonymous referees for their careful reading and helpful comments.

\section{References}

[1] J. F. Andrews. A mathematical model for the continuous culture of microorganisms utilizing inhibitory substrates. Biotechnol. Bioeng., 10 (1968), 707-723.

[2] R. Arditi, J.-M. Abillon, J. V. Da Silva. The effect of a time-delay in a predator-prey model. Math. Biosci., 33 (1977), 107-120.

[3] M. Baptistini, P. Táboas. On the stability of some exponential polynomials. Math. Anal. Appl., 205 (1997), 259-272.

[4] M. S. Bartlett. On theoretical models for competitive and predatory biological systems. Biometrika, 44 (1957), 27-42.

[5] J. R. Beddington, J. G. Cooke. Harvesting from a prey-predator complex. Ecol. Modelling, 14 (1982), 155-177.

[6] R. Bellman, K. L. Cooke. Differential-difference equations. Academic Press, New York, 1963.

[7] E. Beretta, Y. Kuang. Convergence results in a well-known delayed predator-prey system. J. Math. Anal. Appl., 204 (1996), 840-853.

[8] E. Beretta, Y. Kuang. Global analysis in some delayed ratio-dependent predator-prey systems. Nonlinear Anal., 32 (1998), 381-408.

[9] E. Beretta, Y. Kuang. Geometric stability switch crteria in delay differential equations with delay dependent parameters. SIAM J. Math. Anal., 33(2002), 1144-1165.

[10] F. G. Boes. Stability criteria for second-order dynamical systems involving several time delays. SIAM J. Math. Anal., 26 (1995), 1306-1330.

[11] F. Brauer. Stability of some population models with delay. Math. Biosci., 33 (1977), 345-358.

[12] F. Brauer. Characteristic return times for harvested population models with time lag. Math. Biosci., 45 (1979), 295-311. 
[13] F. Brauer. Absolute stability in delay equations. J. Differential Equations, 69 (1987), 185191.

[14] F. Brauer, A. C. Soudack. Stability regions and transition phenomena for harvested predatorprey systems. J. Math. Biol., 7 (1979), 319-337.

[15] F. Brauer, A. C. Soudack. Stability regions in predator-prey systems with constant-rate prey harvesting. J. Math. Biol., 8 (1979), 55-71.

[16] F. Brauer, A. C. Soudack. Coexistence properties of some predator-prey systems under constant rate harvesting and stocking. J. Math. Biol., 12 (1981), 101-114.

[17] M. Brelot. Sur le problème biologique héréditaiare de deux especès dévorante et dévorée. Ann. Mat. Pura Appl., 9 (1931), 58-74.

[18] A. W. Bush, A. E. Cook. The effect of time delay and growth rate inhibition in the bacterial treatment of wastewater. J. Theoret. Biol., 63 (1976), 385-395.

[19] Y. Cao, H. I. Freedman. Global attractivity in time-delayed predator-prey systems. J. Austral. Math. Soc. Ser. B, 38 (1996), 149-162.

[20] J. Caperon. Time lag in population growth response of isochrysis galbana to a variable nitrate environment. Ecology, 50 (1969), 188-192.

[21] Y.-S. Chin. Unconditional stability of systems with time-lags. Acta Math. Sinica, 1 (1960), 125-142.

[22] K. L. Cooke, Z. Grossman. Discrete delay, distributed delay and stability switches. J. Math. Anal. Appl., 86 (1982), 592-627.

[23] K. L. Cooke, P. van den Driessche. On zeros of some transcendental equations. Funkcialaj Ekvacioj, 29 (1986), 77-90.

[24] J. M. Cushing. Integrodifferential Equations and Delay Models in Population Dynamics. Springer-Verlag, Heidelberg, 1977.

[25] J. M. Cushing. Stability and maturation periods in age structured populations. In "Differential Equations and Applications in Ecology, Epidemics, and Population Problems", S. Busenberg and K. L. Cooke (Eds.), Academic Press, New York, 1981, pp. 163-182.

[26] J. M. Cushing, M. Saleem. A predator prey model with age structure. J. Math. Biol., 14 (1982), 231-250. Erratum: 16 (1983), 305.

[27] G. Dai, M. Tang. Coexistence region and global dynamics of a harvested predator-prey system. SIAM J. Appl. Math., 58 (1998), 193-210.

[28] L. S. Dai. Nonconstant periodic solutions in predator-prey systems with continuous time delay. Math. Biosci., 53 (1981), 149-157. 
[29] R. Datko. A procedure for determination of the exponential stability of certain differential difference equations. Quart. Appl. Math., 36 (1978), 279-292.

[30] J. Dieudonné. Foundations of modern analysis. Academic Press, New York, 1960.

[31] B. Ermentrout. Simulating, analyzing, and animating dynamical systems: a guide to XPPAUT for researchers and students. SIAM, Philadelphia, 2002.

[32] T. Faria. Stability and bifurcation for a delayed predator-prey model and the effect of diffusion. J. Math. Anal. Appl., 254 (2001), 433-463.

[33] T. Faria, L. T. Magalhães. Normal forms for retarded functional differential equations and applications to Bogdanov-Takens singularity. J. Differential Equations, 122 (1995), 201-224.

[34] T. Faria, L. T. Magalhães. Normal forms for retarded functional differential equations with parameters and applications to Hopf bifurcations. J. Differential Equations, 122 (1995), 181200.

[35] A. Farkas, M. Farkas, G. Szabó. Multiparameter bifurcation diagrams in predator-prey models with time lag. J. Math. Biol., 26 (1988), 93-103.

[36] H. I. Freedman. Deterministic Mathematical Models in Population Ecology. HIFR Consulting Ltd., Edmonton, 1987.

[37] H. I. Freedman, K. Gopalsamy. Nonoccurence of stability switching in systems with discrete delays. Canad. Math. Bull., 31 (1988), 52-58.

[38] H. I. Freedman, V. S. H. Rao. The tradeoff between mutual interference and time lags in predator-prey systems. Bull. Math. Biol., 45 (1983), 991-1004.

[39] H. I. Freedman, V. S. H. Rao. Stability criteria for a system involving two time delays. SIAM J. Appl. Anal., 46 (1986), 552-560.

[40] H. I. Freedman, G. S. K. Wolkowicz. Predator-prey systems with group defence: The paradox of enrichment revisited. Bull. Math. Biol., 48 (1986), 493-508.

[41] N. S. Goel, S. C. Maitra, E. W. Montroll. On the Volterra and other nonlinear models of interacting populations. Rev. Modern Phys., 43 (1971), 231-276.

[42] K. Gopalsamy. Harmless delay in model systems. Bull. Math. Biol., 45 (1983), 295-309.

[43] K. Gopalsamy. Delayed responses and stability in two-species systems. J. Austral. Math. Soc. Ser. B, 25 (1984), 473-500.

[44] K. Gopalsamy. Stability and Oscillations in Delay Differential Equations of Population Dynamics. Kluwer Academic, Dordrecht, 1992. 
[45] S. Gourley, Y. Kuang. A stage structured predator-prey model and its dependence on maturation delay and death rate. J. Math. Biol., 49 (2004), 188-200.

[46] J. K. Hale, E. F. Infante, F.-S. P. Tsen. Stability in linear delay equations. J. Math. Anal. Appl., 105 (1985), 533-555.

[47] J. K. Hale, S. M. Verduyn Lunel. Introduction to functional differential equations. SpringerVerlag, New York, 1993.

[48] B. D. Hassard, N. D. Kazarinoff, Y.-H. Wan. Theory and applications of Hopf bifurcation. Cambridge University Press, London, 1981.

[49] A. Hastings. Age-dependent predation is not a simple process: I. continuous time models. Theoret. Pop. Biol., 23 (1983), 347-362.

[50] A. Hastings. Delays in recruitment at different trophic levels: effects on stability. J. Math. Biol., 21 (1984), 35-44.

[51] X.-Z. He. Stability and delays in a predator-prey system. J. Math. Anal. Appl., 198 (1996), 355-370.

[52] X.-Z. He. The Lyapunov functionals for delay Lotka-Volterra-type models. SIAM J. Appl. Math., 58 (1998), 1222-1236.

[53] W. L. Hogarth, J. Norbury, I. Cunning, K. Sommers. Stability of a predator-prey model with harvesting. Ecol. Modelling, 62 (1992), 83-106.

[54] W. Huang. Algebraic criteria on the stability of the zero solutions of the second order delay differential equations. J. Anhui University, (1985), 1-7.

[55] J. A. Hutchings, R. A. Myers. What can be learned from the collapse of a renewable resource? Atlantic code, Gadus morhua, of Newfoundland and Labrador. Can. J. Fish. Aquat. Sci., 51 (1994), 2126-2146.

[56] Y. Kuang. Delay differential equations with applications in population dynamics. Academic Press, New York, 1993.

[57] Y. A. Kuznetsov. Elements of applied bifurcation theory. Applied Mathematical Sciences 112, Springer-Verlag, New York, 1995.

[58] S. Liu, L. Chen, R. Agarwal. Recent progress on stage-structured population dynamics. Math. Computer Model.,36 (2002), 1319-1360.

[59] Z. Liu, R. Yuan. Stability and bifurcation in a delayed predator-prey system with BeddintonDeAngelis functional response. J. Math. Anal. Appl., 296 (2004), 521-537.

[60] Z. Lu, W. Wang. Global stability for two-species Lotka-Volterra systems with delay. J. Math. Anal. Appl., 208 (1997), 277-280. 
[61] Z. Ma. Stability of predation models with time delay. Applicable Anal., 22 (1986), 169-192.

[62] J. M. Mahaffy. A test for stability of linear differential delay equations. Quart. Appl. Math., 40 (1982), 193-202.

[63] A. Martin, S. Ruan. Predator-prey models with delay and prey harvesting. J. Mathematical Biology, 43 (2001), 247-267.

[64] R. M. May. Time delay versus stability in population models with two and three trophic levels. Ecology, 4 (1973), 315-325.

[65] N. MacDonald. Time lags in biological models. Springer-Verlag, Heidelberg, 1978.

[66] R. A. Myers, J. A. Hutchings, N. J. Barrowman. Why do fish stocks collapse? The example of cod in Atlantic Canada. Ecol. Appl., 7 (1997), 91-106.

[67] R. A. Myers, B. Worm. Rapid worldwide depletion of large predatory fish communities. Nature, 423 (2003), 280-283.

[68] M. R. Myerscough, B. F. Gray, W. L. Hogarth, J. Norbury. An analysis of an ordinary differential equation model for a two-species predator-prey system with harvesting and stocking. J. Math. Biol., 30 (1992), 389-411.

[69] S. Nakaoka, Y. Saito, Y. Takeuchi. Stability, delay, and chaotic behavior in a Lotka-Volterra predator-prey system. Math. Biosci. Engineer., 3 (2006), 173-187.

[70] L. Nunney. The effect of long time delays in predator-prey systems. Theoret. Pop. Biol., 27 (1985), 202-221.

[71] L. Nunney. Absolute stability in predator-prey models. Theoret. Pop. Biol., 28 (1985), 209232.

[72] Y. Qu, J. Wei. Bifurcation analysis in a time-delay model for prey-predator growth with stage-structure. Nonlinear Dynamics, 49 (2007), 285-294.

[73] G. G. Ross. A difference-differential model in population dynamics. J. Theoret. Biol., 37 (1972), 477-492.

[74] S. Ruan. Absolute stability, conditional stability and bifurcation in Kolmogorov-type predator-prey systems with discrete delays. Quart. Appl. Math., 59 (2001), 159-173.

[75] S. Ruan. Delay differential equations in single species dynamics. In "Delay Differential Equations with Applications," O. Arino, M. Hbid and E. Ait Dads (Eds.), NATO Science Series II: Mathematics, Physics and Chemistry, Vol. 205, Springer, Berlin, 2006, pp. 477-517.

[76] S. Ruan, J. Wei. On the zeros of transcendental functions with applications to stability of delay differential equations. Dynam. Contin. Discr. Impuls. Syst., 10 (2003), 863-874. 
[77] S. Ruan, D. Xiao. Global analysis in a predator-prey system with nonmonotonic functional response. SIAM J, Appl. Math., 61 (2001), 1445-1472.

[78] W. Sokol, J. A. Howell. Kinetics of phenol oxidation by washed cells. Biotechnol. Bioeng., 23 (1980), 2039-2049.

[79] Y. Song, Y. Peng, J. Wei. Bifurcations for a predator-prey system with two delays. J. Math. Anal. Appl., 337 (2008), 466-479.

[80] Y. Song, J. Wei. Local Hopf bifurcation and global periodic solutions in a delayed predatorprey system. J. Math. Anal. Appl., 301 (2005), 1-21.

[81] G. Stépán. Great delay in a predator-prey model. Nonlinear Anal., 10 (1986), 913-929.

[82] P. Táboas. Periodic solutions of a planar delay equation. Proc. Roy. Soc. Edinburgh, 116A (1990), 85-101.

[83] V. Volterra. Variazionie fluttuazioni del numbero d'individui in specie animali conviventi. Mem. Acad. Lincei., 2 (1926), 31-113.

[84] V. Volterra. Lecons sur la théorie mathematique de la lutte pour la vie. Gauthier-Villars, Paris, 1931.

[85] W. Wang, L. Chen. A predator-prey system with stage-structure for predators. Computers Math. Appl., 33 (1997), No. 8, 83-91.

[86] P. J. Wangersky, W. J. Cunningham. Time lag in prey-predator population models. Ecology, 38 (1957), 136-139.

[87] G. S. K. Wolkowicz. Bifurcation analysis of a predator-prey system involving group defence. SIAM J. Appl. Math., 48 (1988), 592-606.

[88] J. Wu. Symmetric functional differential equations and neural networks with memory. Trans. Amer. Math. Soc., 350 (1998), 4799-4838.

[89] J. Xia, Z. Liu, R. Yuan, S. Ruan. The effects of harvesting and time delay on predator-prey systems with Holling type II functional response. SIAM J. Appl. Math. (revised).

[90] D. Xiao, W. Li. Stability and bifurcation in a delayed ratio-dependent predator-prey system. Proc. Edinburgh Math. Soc., 46A (2003), 205-220.

[91] D. Xiao, S. Ruan. Bogdanov-Takens bifurcations in predator-prey systems with constant rate harvesting. Fields Institute Communications, 21 (1999), 493-506.

[92] D. Xiao, S. Ruan. Multiple bifurcations in a delayed predator-prey system with nonmonotonic functional response. J. Differential Equations, 176 (2001), 494-510. 
[93] X.-P. Yan, W.-T. Li. Hopf bifurcation and global periodic solutions in a delayed predatorprey system. Appl. Math. Computat., 177 (2006), 427-445.

[94] T. Zhao, Y. Kuang, H. L. Smith. Global existence of periodic solutions in a class of delayed Gause-type predator-prey systems. Nonlinear Anal., 28 (1997), 1373-1394. 